\title{
MicroRNA-132 enhances transition from inflammation to proliferation during wound healing
}

\author{
Dongqing Li, ${ }^{1}$ Aoxue Wang, ${ }^{1,2}$ Xi Liu, ${ }^{1}$ Florian Meisgen, ${ }^{1}$ Jacob Grünler, ${ }^{3}$ Ileana R. Botusan, ${ }^{3}$ Sampath Narayanan, ${ }^{3}$ Erdem Erikci, ${ }^{4}$ \\ Xi Li, ${ }^{1}$ Lennart Blomqvist, ${ }^{5}$ Lei Du, ${ }^{6}$ Andor Pivarcsi, ${ }^{1}$ Enikö Sonkoly, ${ }^{1}$ Kamal Chowdhury, ${ }^{4}$ Sergiu-Bogdan Catrina, ${ }^{3,7}$ Mona Ståhle, ${ }^{1}$ \\ and Ning Xu Landén \\ 'Molecular Dermatology Research Group, Unit of Dermatology and Venereology, Department of Medicine, Karolinska Institutet (KI), Stockholm, Sweden. ${ }^{2}$ Department of Dermatology, The Second Affiliated \\ Hospital of Dalian Medical University, Dalian, China. ${ }^{3}$ Department of Endocrinology, Metabolism and Diabetes, Karolinska University Hospital, Stockholm, Sweden. ${ }^{4}$ Department of Molecular Cell Biology, \\ Max Planck Institute for Biophysical Chemistry, Cöttingen, Germany. ${ }^{5}$ Department of Medicine Huddinge and ${ }^{6}$ Center for Molecular Medicine, Department of Medicine, KI, Stockholm, Sweden. \\ 'Department of Molecular Medicine and Surgery, Kl, Stockholm, Sweden.
}

\begin{abstract}
Wound healing is a complex process that is characterized by an initial inflammatory phase followed by a proliferative phase. This transition is a critical regulatory point; however, the factors that mediate this process are not fully understood. Here, we evaluated microRNAs (miRs) in skin wound healing and characterized the dynamic change of the miRNome in human skin wounds. miR-132 was highly upregulated during the inflammatory phase of wound repair, predominantly expressed in epidermal keratinocytes, and peaked in the subsequent proliferative phase. TCF- $\beta 1$ and TCF- $\beta 2$ induced miR-132 expression in keratinocytes, and transcriptome analysis of these cells revealed that miR-132 regulates a large number of immune response- and cell cycle-related genes. In keratinocytes, miR-132 decreased the production of chemokines and the capability to attract leukocytes by suppressing the NF-KB pathway. Conversely, miR-132 increased activity of the STAT3 and ERK pathways, thereby promoting keratinocyte growth. Silencing of the miR-132 target heparin-binding EGF-like growth factor (HB-EGF) phenocopied miR-132 overexpression in keratinocytes. Using mouse and human ex vivo wound models, we found that miR-132 blockade delayed healing, which was accompanied by severe inflammation and deficient keratinocyte proliferation. Together, our results indicate that miR-132 is a critical regulator of skin wound healing that facilitates the transition from the inflammatory to the proliferative phase.
\end{abstract}

\section{Introduction}

Wound healing is a fundamental physiological process that maintains the integrity of the skin and includes a series of well-orchestrated biological and molecular events (1). The first stage of wound healing is hemostasis and formation of a provisional wound matrix (up to several hours after injury), which initiates the inflammatory phase (1-3 days afterinjury). Inflammation is essential to clear invading microorganisms and tissue debris, and this phase can be divided into an early phase with neutrophil infiltration and a late phase characterized by the recruitment and differentiation of monocytes. The subsequent proliferative phase (4-21 days after injury) focuses on reepithelialization, formation of granulation tissue, and restoration of the vascular network. The wound repair process ends with the formation of a scar in the remodeling phase, which occurs from day 21 and can last up to 1 year after injury (1). During healing, the transition from the inflammatory to the proliferative phase is a key control point and can determine the outcome of wound healing. Resolution of inflammation at the early phase of healing facilitates the transition of the wound into the subsequent proliferative phase (1). In contrast, excessive and persistent inflammation results in

Authorship note: Dongqing Li and Aoxue Wang contributed equally to this work. Conflict of interest: The authors have declared that no conflict of interest exists. Submitted: September 17, 2014; Accepted: May 14, 2015.

Reference information: J Clin Invest. 2015;125(8):3008-3026. doi:10.1172/JCI79052. failure to enter the proliferative phase, which may lead to a chronic, nonhealing wound $(1,2)$. Chronic wounds affect $0.2 \%$ to $1 \%$ of the population in developed countries, representing a rising health and economic burden for society (1). Thus, identification of novel therapeutic targets for wound healing is a major medical need.

During skin wound healing, epidermal keratinocytes not only participate in reepithelialization, but also play an active and critical role in the innate immune response (2). It has been shown that injury is a major inducer of the release of inflammatory cytokines (e.g., CXC chemokine ligand 1 [CXCL1] and IL-8) by epidermal keratinocytes, which results in rapid recruitment of neutrophils to the wound site and keeps the wound free of infection (3). On the other hand, the immune cells at the wound site also significantly affect the migration and proliferation of keratinocytes by releasing a variety of growth factors and cytokines such as EGF, FGF-2, and TGF- $\beta$ (2). In chronic wounds, the constant inflammatory state results in deregulated differentiation and activation of keratinocytes (4). Therefore, the proper crosstalk between keratinocytes and immune cells is considered crucial for wound repair.

MicroRNAs (miRs) are approximately 22-nt noncoding RNAs that bind to the 3 '-UTR of target mRNAs and result in translational repression or degradation of mRNA (5). Deregulation of miR expression has been shown to be associated with a wide range of diseases. Studies using animal models as well as promising data from recent clinical trials demonstrate that 
modulation of miR expression by administration of specific miR mimics or inhibitors may have beneficial effects in a wide variety of diseases. Therefore, miRs represent promising new therapeutic targets (6).

To develop miR-targeted therapies for skin wounds, the roles of miRs in skin wound healing need to be elucidated. To date, several miRs have been implicated in the biology of skin wound healing using different cell culture and animal model systems (reviewed in ref. 7). However, the dynamic changes in miR expression levels in vivo during wound healing in humans remain unexplored. Here, we reveal marked changes in global miR expression in the inflammatory phase of human skin wound healing and identify miR-132 as a major molecular regulator in human keratinocytes with a critical role in facilitating the transition from inflammation to proliferation.

\section{Results}

Dynamic miRNome changes during the inflammatory phase of human skin wound healing. To study miR expression during human skin wound healing, we created in vivo surgical wounds in the skin of 14 healthy volunteers and collected the tissue around the wounds at different time points after injury that were selected to represent the sequential phases of skin wound healing: hemostasis (0 hour), inflammation (24 hours), proliferation (1 week), and remodeling (1 month) (Supplemental Figure 1A; supplemental material available online with this article; doi:10.1172/JCI79052DS1).

We performed a TaqMan MicroRNA low-density array (TLDA) to compare the expression of 754 miRs in skin from 5 healthy donors at 0 and 24 hours after injury (Figure 1). Unsupervised hierarchical clustering based on miR expression clearly separated 24-hour-old wounds from 0-hour-old wounds (Figure 1). By using the significance analysis of microarrays (SAM) algorithm to analyze the array data, we identified 24 upregulated and 31 downregulated miRs in the 24-hour-old wounds relative to the 0-hour-old wounds (fold change $>1.7$, FDR $<5 \%$ ) (Table 1). Notably, several miRs identified by us in Table 1 have previously been shown to regulate various biological processes related to skin wound healing; these include upregulated miR-15b, miR-16, and miR-20b $(8,9)$ and downregulated miR-210 and miR-146a $(10,11)$.

Expression pattern of miR-132 during skin wound healing. Next, we focused on one of the most upregulated miRs in the inflammatory phase, miR-132 (Table 1), whose function(s) remains unexplored in the skin. We first characterized its expression pattern throughout the entire healing process. By performing quantitative real-time PCR (qRT-PCR) on a set of RNA isolated from wound biopsies from 7 healthy donors at 0 hours, 24 hours, 1 week, and 1 month after injury, we confirmed a significant upregulation (2.8-fold change, $\left.P=1.04 \times 10^{-9}\right)$ of miR-132 expressionin the wounds in the inflammatory phase ( 24 hours) compared with that observed in the 0-hour-old wounds (Figure 2A). Interestingly, expression levels of miR-132 were further increased in the proliferative phase (1 week) relative to those in the inflammatory phase (1.8-fold change, $P=0.04$ ) (Figure 2A). When the wound entered the remodeling phase (1 month), miR-132 expression levels decreased compared with the levels observed in the proliferative phase (0.45-fold change, $P=0.01)$, although they were still higher than in the 0 -hour-old wounds (2.2-fold change, $P=0.01$ ) (Figure $2 \mathrm{~A}$ ).

Table 1. List of the significantly changed miRs in 24-hour-old wounds compared with 0 -hour-old wounds

\begin{tabular}{|c|c|c|c|c|}
\hline & Gene ID & Score (d) & Fold change & FDR (\%) \\
\hline \multirow[t]{24}{*}{ Upregulated } & hsa-miR-142-3p & 5.67 & 6.29 & 0.00 \\
\hline & hsa-miR-31 & 4.78 & 8.89 & 0.00 \\
\hline & hsa-miR-132 & 4.74 & 2.93 & 0.00 \\
\hline & hsa-miR-16 & 4.44 & 2.32 & 0.00 \\
\hline & hsa-miR-93 & 4.20 & 1.77 & 0.00 \\
\hline & hsa-miR-19a & 3.97 & 1.80 & 0.00 \\
\hline & hsa-miR-484 & 3.96 & 1.76 & 0.00 \\
\hline & hsa-miR-150 & 3.95 & 2.45 & 0.00 \\
\hline & hsa-miR-135b & 3.62 & 3.41 & 1.02 \\
\hline & hsa-miR-31-3p & 3.59 & 2.99 & 1.02 \\
\hline & hsa-miR-155 & 3.57 & 2.32 & 1.02 \\
\hline & hsa-miR-886-3p & 3.48 & 2.46 & 1.02 \\
\hline & hsa-miR-451 & 3.37 & 18.53 & 1.53 \\
\hline & hsa-miR-223 & 3.28 & 63.33 & 1.53 \\
\hline & hsa-miR-652 & 3,28 & 3,10 & 1,53 \\
\hline & hsa-miR-25 & 3.16 & 2.01 & 1.53 \\
\hline & hsa-miR-340 & 3.15 & 3.55 & 1.53 \\
\hline & hsa-miR-223-5p & 3.10 & 11.80 & 1.53 \\
\hline & hsa-miR-144-5p & 3.08 & 19.43 & 1.53 \\
\hline & hsa-miR-20b & 2.88 & 7.52 & 1.53 \\
\hline & hsa-miR-486 & 2.73 & 20.05 & 1.71 \\
\hline & hsa-miR-425-5p & 2.46 & 2.79 & 3.48 \\
\hline & hsa-miR-15b & 2.37 & 4.68 & 4.14 \\
\hline & hsa-miR-142-5p & 2.33 & 3.84 & 4.14 \\
\hline \multirow[t]{31}{*}{ Downregulated } & hsa-miR-539 & -1.98 & 0.53 & 4.14 \\
\hline & hsa-miR-101 & -2.01 & 0.53 & 3.48 \\
\hline & hsa-miR-27a & -2.19 & 0.57 & 2.02 \\
\hline & hsa-miR-1271 & -2.22 & 0.43 & 2.02 \\
\hline & hsa-miR-455 & -2.33 & 0.50 & 2.00 \\
\hline & hsa-miR-411 & -2.45 & 0.43 & 2.00 \\
\hline & hsa-miR-328 & -2.53 & 0.54 & 2.00 \\
\hline & hsa-miR-409-3p & -2.57 & 0.54 & 2.00 \\
\hline & hsa-miR-200a & -2.66 & 0.53 & 2.00 \\
\hline & hsa-miR-23a & -2.85 & 0.46 & 2.00 \\
\hline & hsa-miR-199a-3p & -2.91 & 0.55 & 2.00 \\
\hline & hsa-miR-214-5p & -2.93 & 0.41 & 2.00 \\
\hline & hsa-miR-221 & -2.97 & 0.57 & 2.00 \\
\hline & hsa-miR-210 & -3.11 & 0.49 & 1.55 \\
\hline & hsa-miR-365 & -3.15 & 0.57 & 1.55 \\
\hline & hsa-miR-99a & -3.18 & 0.45 & 1.55 \\
\hline & hsa-miR-27b & -3.25 & 0.53 & 1.15 \\
\hline & hsa-miR-149 & -3.36 & 0.39 & 1.15 \\
\hline & hsa-miR-23b & -3.46 & 0,53 & 1,15 \\
\hline & hsa-miR-152 & $-3,52$ & 0.52 & 1.15 \\
\hline & hsa-miR-127 & -3.83 & 0.48 & 0.00 \\
\hline & hsa-let-7c & -4.03 & 0.56 & 0.00 \\
\hline & hsa-miR-100 & -4.03 & 0.59 & 0.00 \\
\hline & hsa-miR-146a & -4.22 & 0.58 & 0.00 \\
\hline & hsa-miR-214 & -4.39 & 0.30 & 0.00 \\
\hline & hsa-miR-193b & -5.97 & 0.55 & 0.00 \\
\hline & hsa-miR-196b & -7.04 & 0.51 & 0.00 \\
\hline & hsa-miR-218 & -7.40 & 0.41 & 0.00 \\
\hline & hsa-miR-139-5p & -8.21 & 0.59 & 0.00 \\
\hline & hsa-miR-489 & -8.81 & 0.24 & 0.00 \\
\hline & hsa-miR-574-3p & -13.43 & 0.44 & 0.00 \\
\hline
\end{tabular}


Using qRT-PCR, we also analyzed the expression of primary miR-132 transcripts (pri-miR-132), from which mature miR-132 is processed, in the same wound biopsies. We found that expression levels of pri-miR-132 in the wounds were upregulated after 1 week and then decreased 1 month after wounding (Figure 2B). The expression of pri-miR-132 positively correlated $(R=0.59$, $P=0.004$ ) with the expression of mature miR-132 (Supplemental Figure 1B). These data indicate that regulation of miR-132 expression in wounded skin may occur at both the transcriptional level and during processing of the primary transcripts.

In order to reveal which cell type(s) are primarily responsible for the changes in miR-132 expression levels during wound healing, we performed in situ hybridization (ISH) with miR-132-specific locked nucleic acid-modified (LNA-modified) probes on skin wound sections obtained from 7 healthy individuals (Figure 2C). The miR-132 signal was mainly detected in epidermal keratinocytes. In accordance with our PCR data, miR-132 expression levels were low in the 0-hour-old wounds, but gradually increased in the wounds collected 12 hours after injury. In the proliferative phase (7 days), the expression of miR-132 reached its peak and subsequently diminished 14 days after wounding (Figure 2C). Taken together, the results of our qRT-PCR and ISH analyses demonstrate that miR-132 expression levels are tightly regulated in epidermal keratinocytes at the wound edge, increase during the inflammatory phase, and peak during the proliferative phase.

miR-132 expression is induced by TGF- $\beta$ in keratinocytes. To understand the mechanism(s) regulating miR-132 expression in keratinocytes at the wound edge, we systematically surveyed the effect of factors and conditions previously shown to regulate skin wound healing on miR-132 expression in keratinocytes. Human primary keratinocytes were treated with cytokines (IL-1 $\alpha$, IL-1 $\beta$, IL-4, IL-6, IL-10, IL-13, IL-17a, IL-17f, IL-20, IL-21, IL-22, IFN- $\gamma$, TNF- $\alpha$, TGF- $\beta 1$, and TGF- $\beta 2$ ), growth factors (EGF and KGF), and keratinocyte differentiation-driving factors $(1.5 \mathrm{mM} \mathrm{CaCl}$ and 12-O-tetradecanoylphorbol-13-acetate [TPA]), and miR-132 expression levels were analyzed by qRT-PCR (Supplemental Figure 2). We found that miR-132 expression was significantly increased only by TGF- $\beta 1$ and TGF- $\beta 2$ (Figure $2 \mathrm{D}$ ), but not by the other factors (Supplemental Figure 2). It has been shown that TGF- $\beta$ is upregulated in skin wounds and plays a crucial role in regulating keratinocyte migration and proliferation during reepithelialization (12). Here, we measured the expression of TGF- $\beta$ in human wound biopsies and confirmed that both TGF- $\beta 1$ (Figure 2E) and TGF- $\beta 2$ (Figure 2F) were upregulated in skin wounds, although with different kinetics. Moreover, we show that TGF- $\beta$ also induced the expression of pri-miR-132 in keratinocyte culture, indicating that TGF- $\beta$ regulates miR-132 gene transcription (Figure $2 \mathrm{G}$ ). Importantly, when the TGF- $\beta$ receptor was specifically blocked by a chemical inhibitor, SB431542, the inducible effect of TGF- $\beta$ on miR-132 expression was suppressed (Figure $2 \mathrm{H}$ ).

To investigate whether TGF- $\beta$ regulates miR-132 expression directly or indirectly (e.g., through induction of other secreted mediators), we collected conditioned medium from keratinocytes treated with TGF- $\beta$ for 48 hours, and the induced expression of miR-132 was confirmed in these cells by qRT-PCR (Supplemental Figure $3 \mathrm{~A})$. We incubated the conditioned medium with untreated

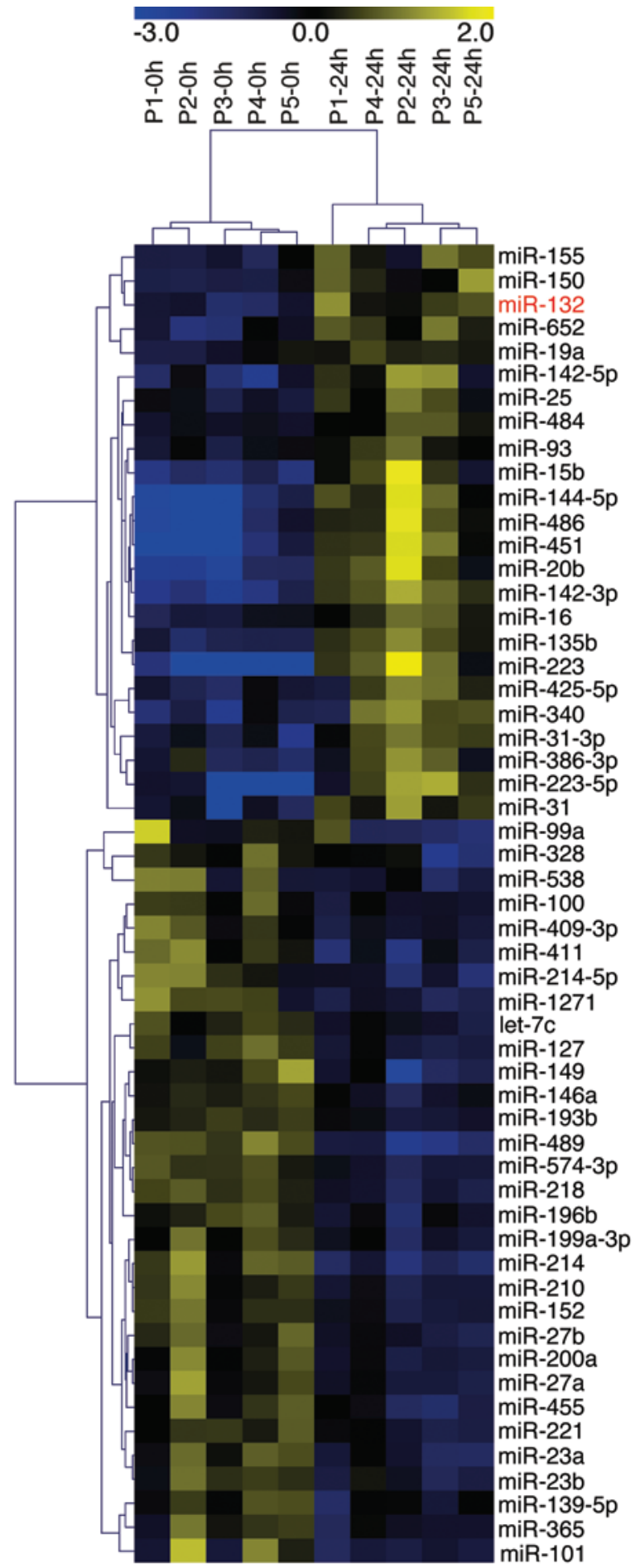

Figure 1. Dynamic change of miR expression in the inflammatory phase of human skin wound healing. miR expression profiling for skin from healthy donors $(n=5)$ at 0 hours and 24 hours after injury. Heatmap illus trating levels of significantly changed miRs (fold change $>1.7$, FDR $<5 \%$ ). Color intensity is scaled within each row so that the highest expression value corresponds to bright yellow and the lowest to bright blue. 

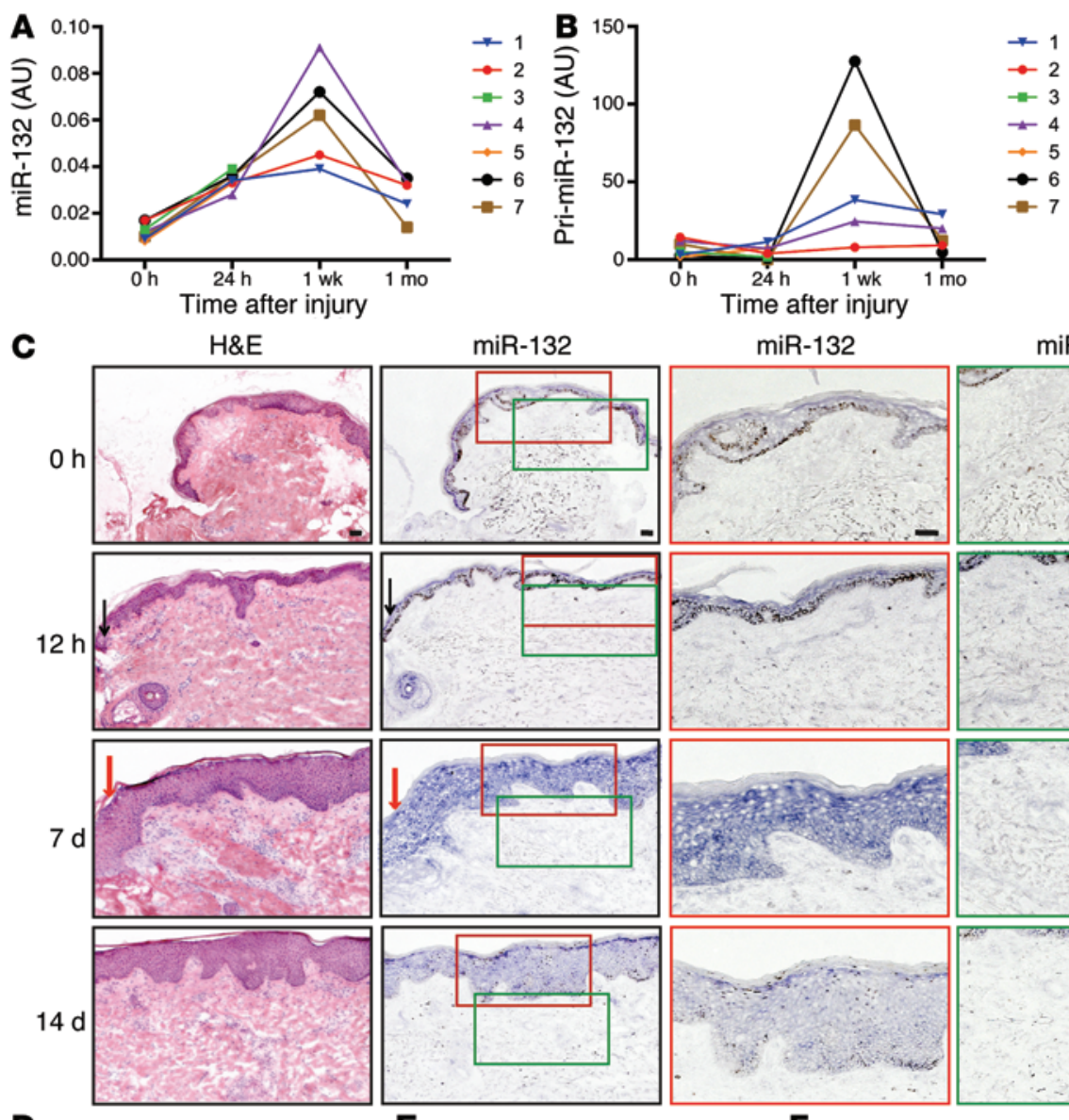

$\operatorname{miR}-132$
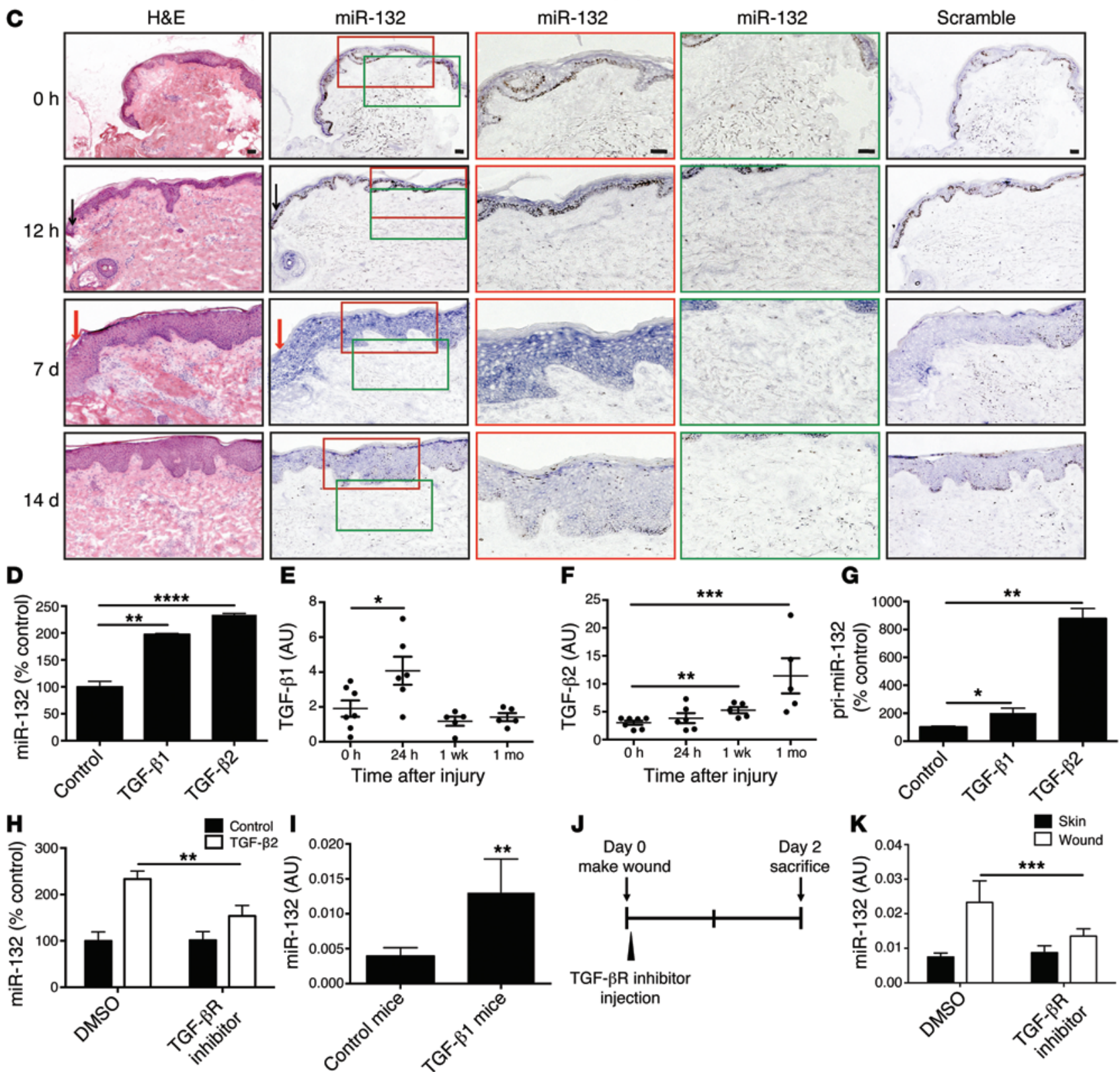

Figure 2. Expression and regulation of miR-132 in wounds. Expression levels of miR-132 (A) and primary miR-132 (pre-miR-132) transcripts (B) in wound biopsies from healthy donors $(n=7)$ at 0 hours, 24 hours, 1 week, and 1 month after injury were analyzed by qRT-PCR. (C) ISH was performed on wound biopsies from healthy donors $(n=7)$ at 0 hours, 12 hours, 7 days, and 14 days after injury. The first column: H\&E staining; second-fourth columns: ISH using a miR-132-specific probe; fifth column: ISH using a scrambled probe. Blue-purple color indicates miR-132 expression. Black arrows demarcate wound edges, while red ones point to newly formed epidermis. Scale bars: $50 \mu \mathrm{m}$. (D) qRT-PCR analysis of miR-132 expression in keratinocytes treated with TGF- $\beta 1$ or TGF- $\beta 2$ for 48 hours $(n=3)$. qRT-PCR analysis of TCF- $\beta 1(E)$ and TGF- $\beta 2(\mathbf{F})$ expression in wound biopsies from healthy donors $(n=7)$. (G) pri-miR-132 expression was analyzed in keratinocytes treated with TGF- $\beta 1$ or TGF- $\beta 2(n=3)$. (H) The TCF- $\beta$ receptor inhibitor SB431542 was applied 15 minutes before adding TGF- $\beta 2$ to keratinocytes, and miR-132 expression was analyzed 48 hours later $(n=3)$. (I) qRT-PCR analysis of miR-132 expression in the skin of TCF- $\beta 1$ Tg mice $(n=3)$ and control littermates $(n=3)$. (J) SB431542 or DMSO was injected into the wound edges of mice (5 versus 5) immediately after wounding. (K) qRT-PCR analysis of miR-132 expression in intact skin and in 2-day-old wounds $(n=5)$. ${ }^{*} P<0.05,{ }^{*} P<0.01,{ }^{* *} P<0.001$, and ${ }^{* * *} P<0.0001$ by Student's $t$ test. TGF- $\beta R$, TCF- $\beta$ receptor. 
keratinocytes for 6 to 48 hours (Supplemental Figure 3B). The levels of miR-132 expression in the recipient keratinocytes were unchanged, which excludes the possibility that the inducible effect of TGF- $\beta$ on miR-132 expression is mediated by other soluble factors secreted by keratinocytes after TGF- $\beta$ treatment.

To investigate whether miR-132 is regulated by TGF- $\beta$ in vivo, we measured miR-132 expression in the skin of K5.TGF- $\beta 1 \mathrm{Tg}$ mice, which overexpress TGF- $\beta 1$ in an epidermis-specific manner (13, 14). qRT-PCR results showed that miR-132 expression levels were increased 3.3-fold ( $P=0.002)$ in the skin of K5.TGF- $\beta 1$ mice compared with the levels detected in their WT littermates (Figure 2I). Moreover, we found that blocking TGF- $\beta$ receptors in vivo by injecting SB431542 into the wound edges in C57BL/6N mice abolished injury-induced miR-132 expression in vivo (Figure 2, J and K). Collectively, our results suggest that increased levels of TGF- $\beta$ in wounds contribute to the upregulation of miR-132 in keratinocytes at the wound edge.

Characterization of the transcriptome regulated by miR-132 in keratinocytes. To study the role(s) of miR-132 in the cellular processes involved in wound healing, we overexpressed or inhibited endogenous miR-132 by transfecting synthetic miR-132 precursor RNA (pre-miR-132) or miR-132-specific inhibitor (anti-miR-132) into human primary keratinocytes, respectively. The overexpression and inhibition of miR-132 in keratinocytes were confirmed by qRT-PCR (Supplemental Figure 4, A and B) and by a luciferase reporter assay using a synthetic miR-132 target as the sensor (Supplemental Figure 4C).

We first performed global transcriptomic analysis of human primary keratinocytes upon overexpression of miR-132 using Affymetrix arrays, which identified 489 genes whose levels were significantly (fold-change $\geq 1.2$, FDR $<5 \%$ ) regulated by miR-132 in keratinocytes (Figure $3 \mathrm{~A}$ and Supplemental Table 1). Using the TargetScan target prediction algorithm (15), we found that 31 of 210 genes downregulated by miR-132 contained potential binding sites for miR-132, whereas only 1 of 279 genes upregulated by miR-132 was predicted to be targeted by miR-132 (Figure $3 \mathrm{~A})$. We also examined the correlation between the presence of miR-132-binding sites and the degree of mRNA regulation and found that mRNAs containing miR-132-binding sites displayed greater reductions in expression levels than did any of the genes in this array (Figure 3B). Moreover, according to the DIANA-mirExTra algorithm (16), we found that miR-132-binding sites were more enriched in the genes downregulated by miR-132 than in those upregulated or not changed by miR-132 (Figure 3C). Altogether, these results suggest that this transcriptomic profiling is specific and sensitive for detecting the genes regulated by miR- 132 .

Next, we performed gene ontological analysis to classify the genes regulated by miR-132 according to their known functions (17). The 10 most enriched biological processes for the genes downregulated and upregulated by miR-132 in keratinocytes are listed in Figure 3, D and E, respectively. Interestingly, for the genes downregulated by miR-132, immune response-related genes were the most enriched (Figure 3D); for the genes upregulated by miR-132, all enriched processes were related to the cell cycle (Figure 3E). These results prompted us to further explore the potential role(s) of miR-132 in the immune response and in the proliferation of keratinocytes.
miR-132 suppresses keratinocyte chemokine production by regulating the NF- $\kappa B$ pathway. Keratinocytes have important roles in the activation of innate immune responses during wound healing. They can produce inflammatory chemokines to attract immune cells migrating into the wound, which in turn fight infections and contribute to wound healing. Since the results of the transcriptomic analysis revealed a strong effect of miR-132 on immune-associated cellular functions, we analyzed its effect on chemokine production by keratinocytes at both the basal level and under inflammatory conditions, which were mimicked by treatment with TNF- $\alpha$ or IL-1 $\beta$. qRT-PCR analysis of chemokine expression showed that miR-132 overexpression suppressed mRNA levels of IL8, CXCL5, CXCL1, chemokine (C-C motif) ligand 20 (CCL2O), IL1A, IL1B, and TNF in keratinocytes under both basal and inflammatory conditions (Figure 4A, Supplemental Figure 5A, and Supplemental Figure 6). Consistently, the amount of IL-8, CCL20, and CXCL5 secreted into the culture medium was also reduced by pre-miR-132, as shown by ELISA (Supplemental Figure 5B). In accordance with this finding, inhibition of endogenous miR-132 increased the production of these chemokines by keratinocytes (Supplemental Figure 5C).

As functional readouts of miR-132 regulated chemokine and cytokine expression, we measured the endothelial cell-activating and leukocyte-attracting capacity of keratinocytes. The first step of recruitment of leukocytes to the injured skin is the attachment of circulating cells to vascular endothelial cells, which is mediated by cell-adhesion molecules such as vascular cell adhesion protein 1 (VCAM1) and E-selectin (SELE) (18). We measured the expression of these 2 genes in HUVECs incubated with conditioned medium from keratinocytes overexpressing miR-132 and demonstrated that miR-132 decreased the capacity of keratinocytes to induce the expression of these cell-adhesion molecules in endothelial cells (Figure 4B), which is in line with the evidence showing miR-132-mediated downregulation of CXCL1, CXCL5, and IL-8, the chemokines important for endothelial cell activation (19). Moreover, we performed chemotaxis assays with neutrophils (Figure 4C) and mononuclear cells (Supplemental Figure 5D) isolated from human peripheral blood using conditioned supernatants from pre-miR-132-treated keratinocytes. It has been shown that CXCL1, CXCL5, and CXCL8 attract neutrophils, whereas CCL20 attracts mononuclear cells (19). Accordingly, we found that supernatants from keratinocytes with increased miR-132 expression attracted fewer neutrophils and mononuclear cells compared with medium from control-treated cells. Altogether, these results suggest that increased miR-132 expression levels suppress inflammation during wound healing by reducing chemokine and cytokine production by keratinocytes, which results in decreased leukocyte recruitment.

We next aimed to identify the signaling pathway(s) mediating the antiinflammatory effect of miR-132 on keratinocytes. Among the transcripts significantly regulated by miR-132 in keratinocytes, several are known to be regulated by the NF- $\mathrm{B}$ pathway (e.g., IL8, CXCL5, CCL2O, IL1B, and CXCL1) (Supplemental Table 1). We therefore performed gene set enrichment analysis (GSEA) (20) to evaluate enrichment of the known NF- $\mathrm{BB}$ pathway target genes in the microarray data (21). The results of this analysis demonstrated that NF- $\mathrm{BB}$ pathway target genes 
A

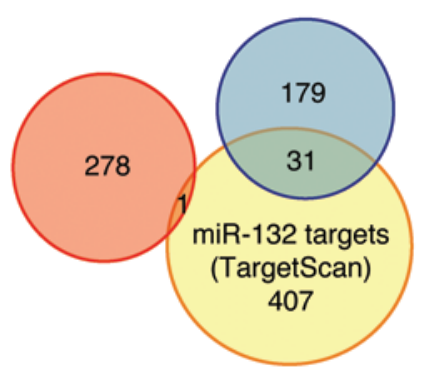

D
B

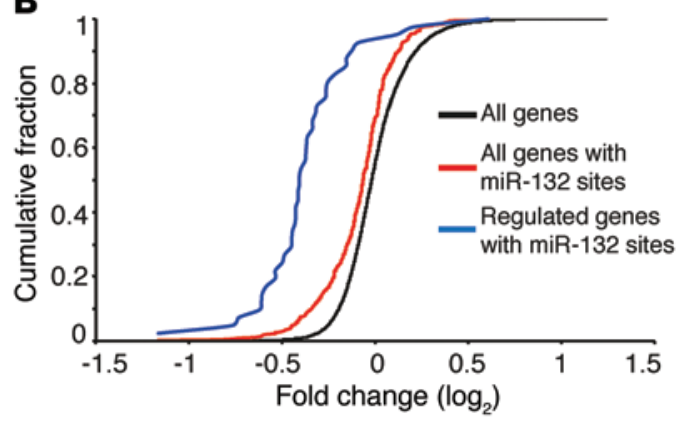

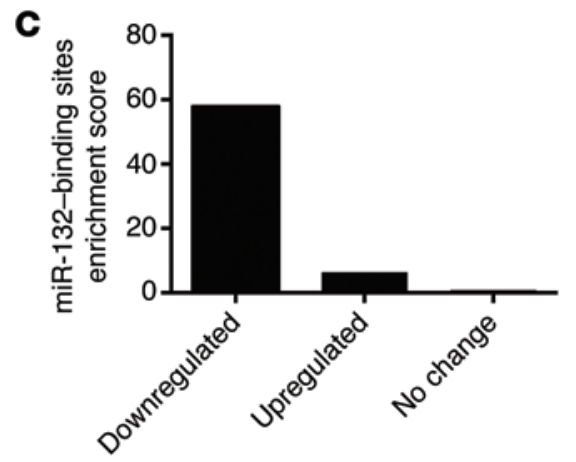

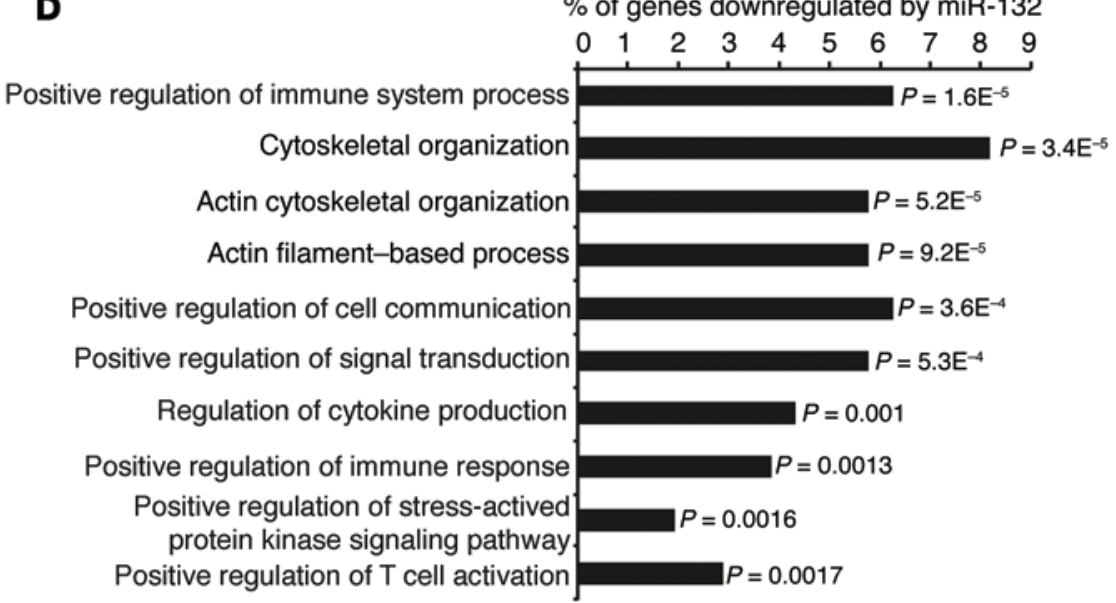

$\mathbf{E}$

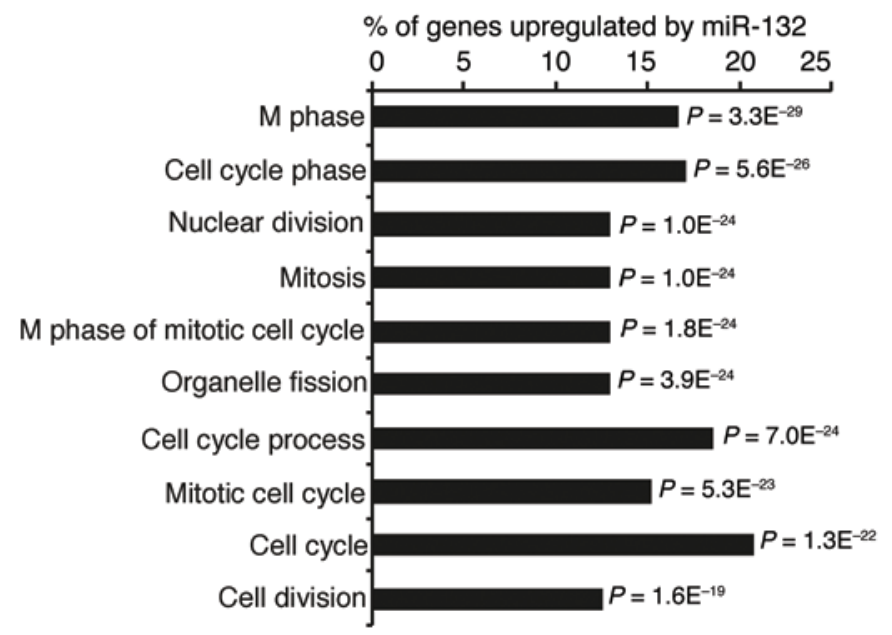

Figure 3. Transcriptomic analysis of keratinocytes overexpressing miR-132. (A) Venn diagram depicting the number of genes significantly up- (red) or downregulated (blue) by miR-132 and TargetScan-predicted miR-132 targets (yellow). (B) Cumulative distribution plots of log ${ }_{2}$-transformed fold changes for genes containing miR-132-binding sites (red), genes with miR-132-binding sites and regulated by miR-132 (blue), and all the genes detected by microarray (black). (C) Microarray data were analyzed using DIANA-mirExTra (http://diana.cslab.ece.ntua.gr/hexamers/). The scores indicate the frequency of miR-132 sites in the 3'UTR of genes down- or upregulated or not changed by miR-132. Top 10 gene ontology terms for the genes down- (D) or upregulated (E) by miR-132 in keratinocytes. $P$ values were determined by Fisher's exact test.

were significantly enriched among the genes downregulated by miR-132, and a negative enrichment score curve was generated $(P<0.05)$ (Figure 4D). This suggests that miR-132 negatively regulates NF- $\kappa \mathrm{B}$ pathway activity. Next, we tested this hypothesis by measuring the effect of pre-miR-132 on NF-kB-dependent luciferase reporter gene expression in keratinocytes. The results of the luciferase assays showed that overexpression of miR-132 suppressed both basal and TNF- $\alpha$-induced luciferase activity, demonstrating that miR-132 is a negative regulator of the NF- $\kappa B$ pathway in keratinocytes (Figure 4E).

Furthermore, we analyzed the effect of miR-132 on the critical steps of NF- $\mathrm{kB}$ signaling. We treated keratinocytes with TNF- $\alpha$, which activated the NF-kB pathway by phosphorylating the inhibitor of NF-кB (ІкB) and subsequently resulted in 

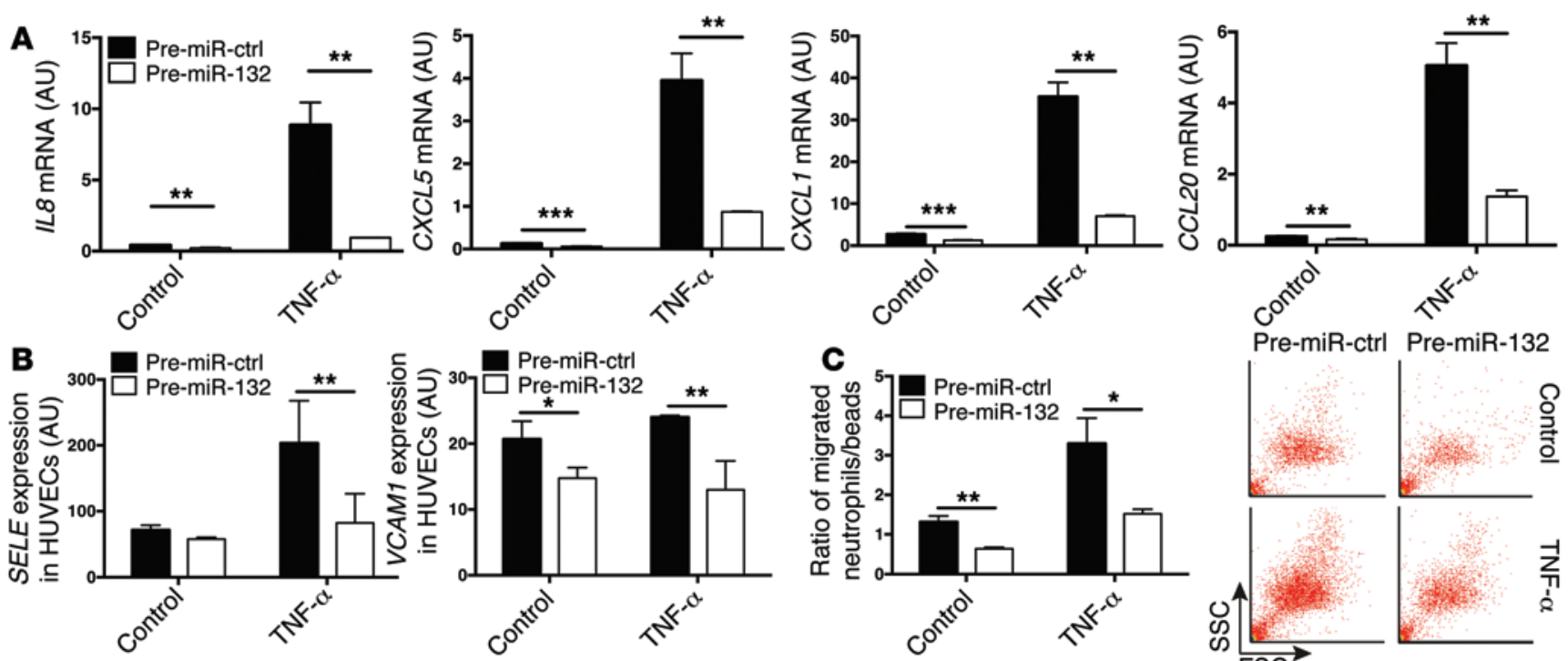

D GSEA, miR-132 overexpression 20,365 genes ranked by fold change
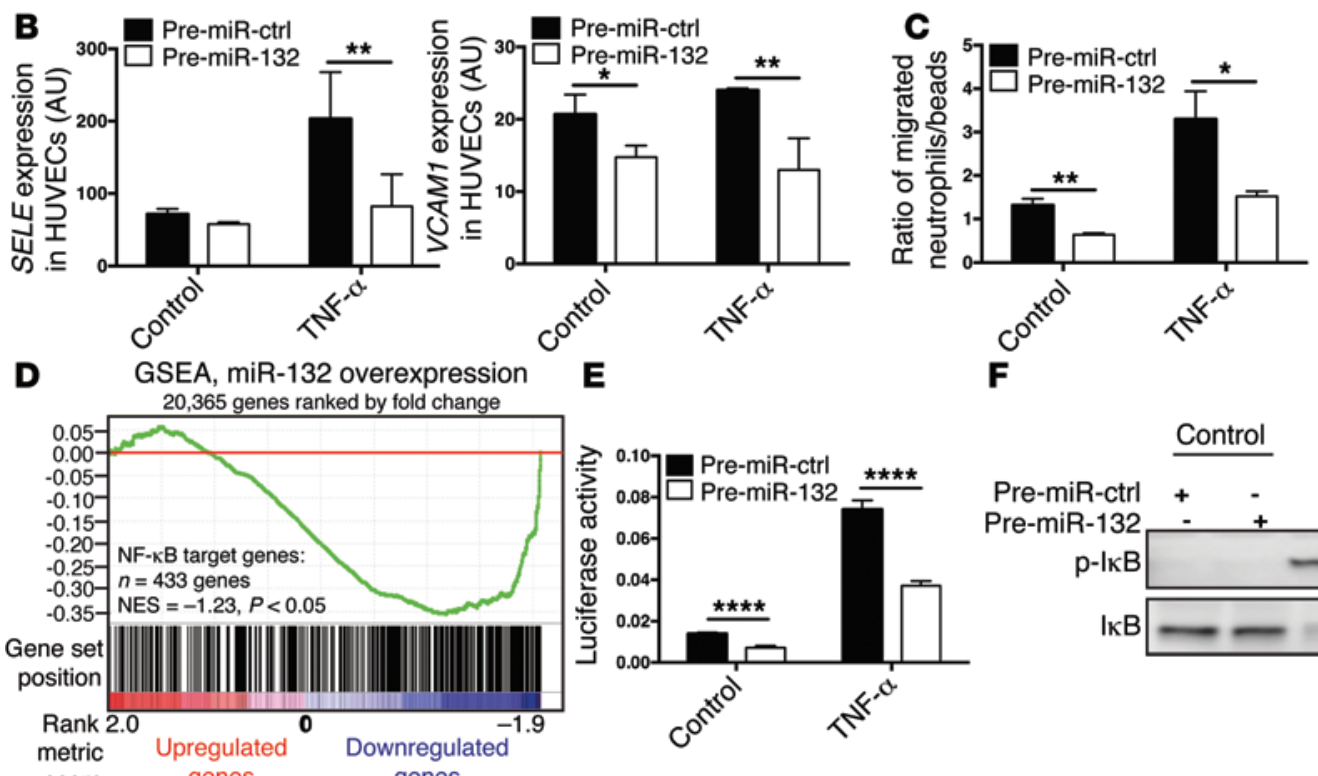

Pre-miR-ctrl Pre-miR-132
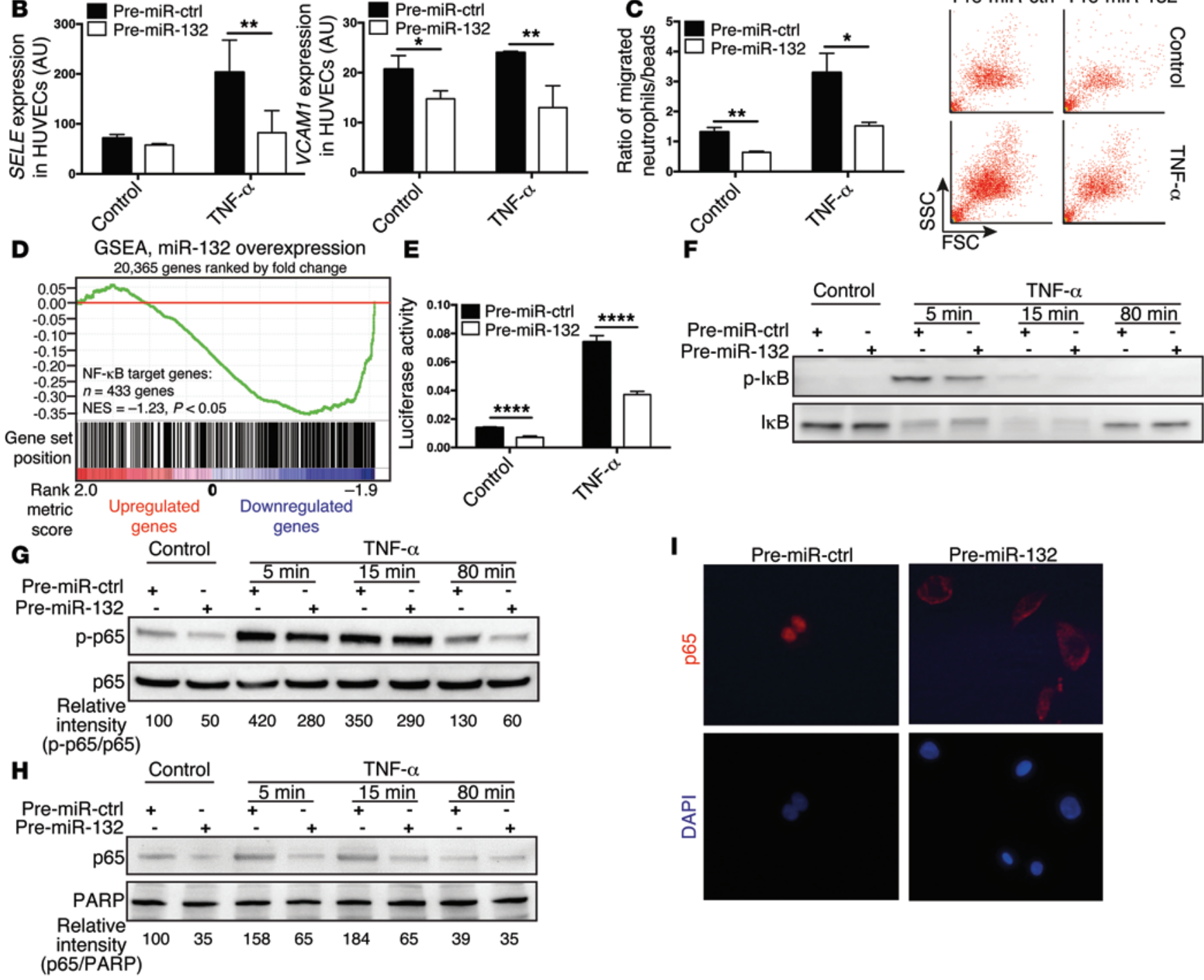

Figure 4. $\mathbf{m i R}-132$ regulates keratinocyte chemokine production by suppressing NF- $\mathrm{B}$ signaling. (A) qRT-PCR analysis of chemokine expression in keratinocytes transfected with pre-miR-132 or pre-miR-Ctrl for 48 hours and then treated with TNF- $\alpha$ for 24 hours $(n=3)$. (B) HUVECs were incubated with conditioned medium from keratinocytes treated as above, and expression of endothelial cell activation markers was analyzed by qRT-PCR $(n=3)$. (C) Human neutrophil chemotaxis toward the conditioned medium from keratinocytes treated as above. Flow cytometric plots show forward scatter/side scatter (FSC/SSC) of the migrated cells. (D) Genes in microarray data were ranked by fold change (pre-miR-132/pre-miR-Ctrl). GSEA eval-

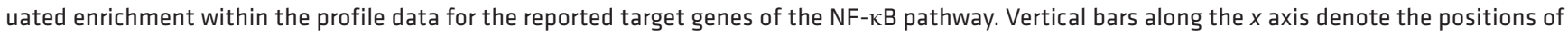
the NF- $\mathrm{KB}$ target genes within the ranked list. NES, normalized enrichment score. (E) Luciferase activity was measured in keratinocytes transfected with NF- $\mathrm{KB}$ reporter and pre-miR-132 or pre-miR-Ctrl for 24 hours and treated with TNF- $\alpha$ for 5 hours $(n=3)$. Keratinocytes transfected with premiR-132 or pre-miR-Ctrl for 48 hours were treated with TNF- $\alpha$ for 5 to 80 minutes. Phosphorylated and total I $\kappa B(F)$ and phosphorylated and total p65 (G) were detected in total protein lysates; 665 was detected in the nuclear lysates by Western blotting; and PARP was detected on the same blots as the loading control (H). (I) Immunostaining of p65 in keratinocytes transfected with pre-miR-132 or pre-miR-Ctrl for 48 hours then treated with TNF- $\alpha$ for 20 minutes. Cells were counterstained using DAPI. ${ }^{*} P<0.05,{ }^{*} P<0.01,{ }^{* *} P<0.001$, and ${ }^{* * * *} P<0.0001$ by Student's $t$ test. 
A

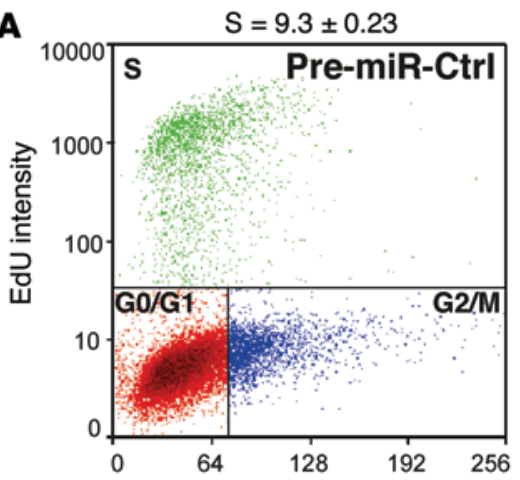

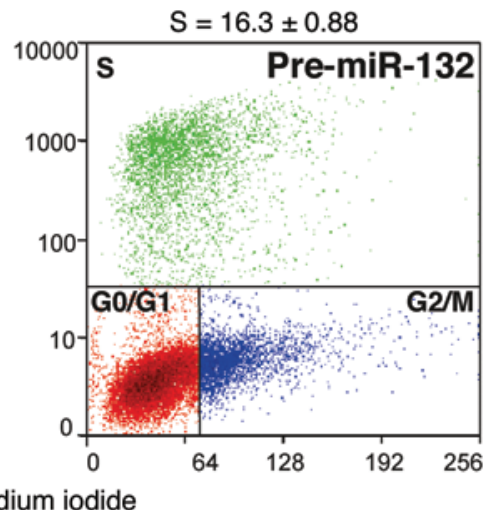

C

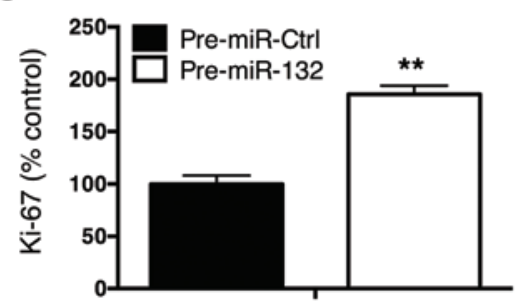

$\mathbf{F}$
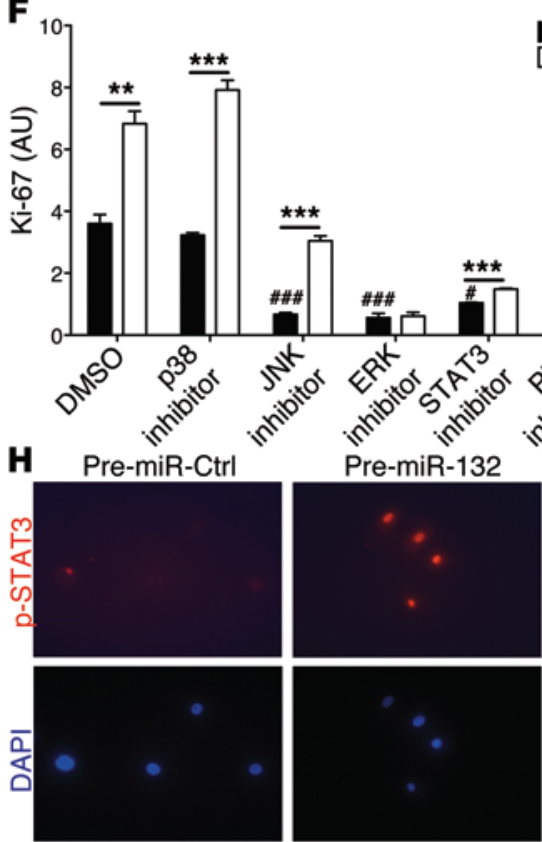

D

Pre-miR-132

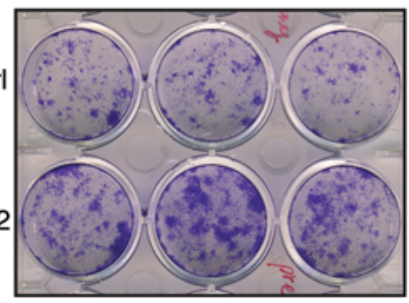

G
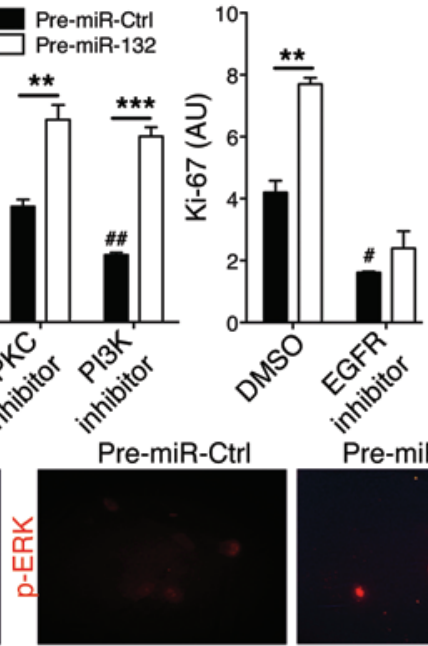

Pre-miR-132

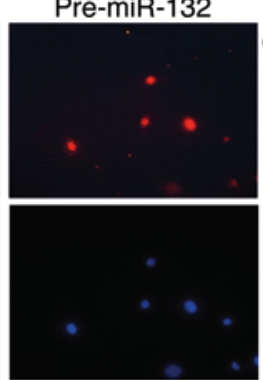

B

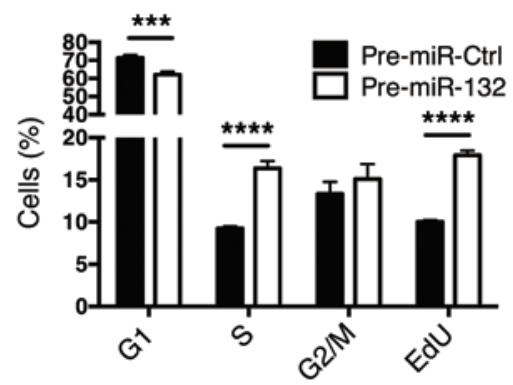

E

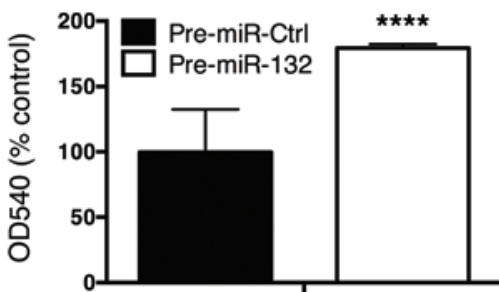

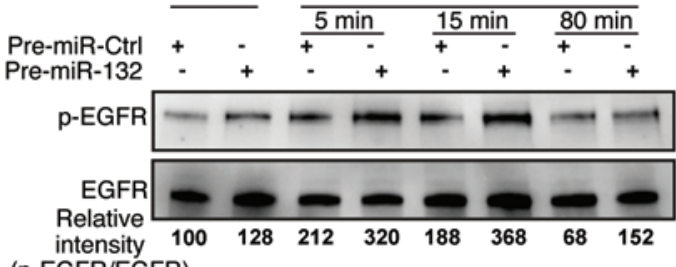

(p-EGFR/EGFR

p-STAT3

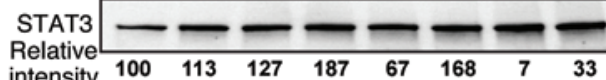

intensity 100

p-STAT3/STAT3)

p-ERK $-\cdots-\infty-$

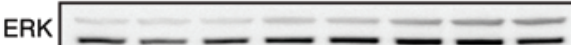

Relative $\begin{array}{lllllllllll}100 & 300 & 900 & 1300 & 1900 & 2060 & 1240 & 1460\end{array}$ intensity

(p-ERK/ERK)

Figure 5. miR-132 promotes keratinocyte growth by increasing EGFR, STAT3, and ERK signaling activity. (A) Primary human keratinocytes were transfected with pre-miR-132 or pre-miR-Ctrl for 48 hours. Cell proliferation and cell cycle progression were measured by EdU labeling and subsequent cell cycle analysis. Representative flow cytometric plots from 4 independent experiments are shown. (B) Percentage of cells in the G1, S, and $\mathrm{G} 2 / \mathrm{M}$ phases of the cell cycle and percentage of EdU+ cells $(n=4)$. (C) Expression of the proliferation marker Ki-67 was analyzed in keratinocytes transfected with pre-miR-132 or pre-miR-Ctrl for 48 hours using qRT-PCR $(n=3)$. (D) Colonies formed by the transfected keratinocytes were stained with crystal violet 8 days after transfection. (E) Crystal violet was dissolved with methanol, and the absorbance value was measured at $540 \mathrm{~nm}$ $(n=6)$. (F) Keratinocytes transfected with pre-miR-132/pre-miR-Ctrl for 24 hours were treated with various inhibitors or DMSO for another 24 hours. $\mathrm{Ki}-67$ was detected by qRT-PCR $(n=3)$. (G) Keratinocytes transfected with pre-miR-132/pre-miR-Ctrl for 48 hours were treated with EGF for 5 to 80 minutes. Phosphorylated and total EGFR, STAT3, and ERK were detected by Western blotting. (H) Immunostaining of p-STAT3 and p-ERK in keratinocytes that were transfected with pre-miR-132/pre-miR-Ctrl for 48 hours, then treated with EGF for 15 minutes. The cells were counterstained with DAPI (blue, nucleus). (B, C, E, and F) ${ }^{* *} P<0.01,{ }^{* *} P<0.001$, and ${ }^{* * * *} P<0.0001$ by Student's $t$ test. ${ }^{*} P<0.05$, \#\# $P<0.01$, and ${ }^{\# \# \#} P<0.001$ in $\mathbf{F}$ for inhibitor-treated cells compared with DMSO-treated cells. 
its degradation. This allowed NF- $\kappa$ B subunits, including p65, to translocate into the nucleus and induce the expression of proinflammatory genes (22). We found that overexpression of miR-132 decreased the level of phosphorylated I $\mathrm{B}$ and its degradation (Figure $4 \mathrm{~F}$ ). In line with this, less p65 was phosphorylated (Figure 4G) and entered nuclei (Figure 4, $\mathrm{H}$ and I) in the keratinocytes transfected with pre-miR-132 compared with those transfected with control oligos. Collectively, these data demonstrate that miR-132 inhibits NF- $\kappa$ B pathway activity and provide an explanation for the mechanism of action of miR-132 in chemokine production in keratinocytes.

miR-132 promotes keratinocyte growth by regulating STAT3 and ERK pathways. The large number of genes related to cell proliferation and cell cycle among the miR-132-regulated genes suggests that miR-132 may be involved in the regulation of keratinocyte growth. To test this, we analyzed the proliferation rate by 5 -ethynyl-2'-deoxyuridine (EdU) incorporation assay for the keratinocytes transfected with pre-miR-132 or control oligos (Figure 5, A and B). Overexpression of miR-132 resulted in a 1.8fold increase $\left(P=1.7 \times 10^{-7}\right)$ in the percentage of EdU-positive cells compared with keratinocytes transfected with control oligos (Figure 5B), indicating that miR-132 increases cell proliferation. Cell cycle distribution analysis revealed that overexpression of miR-132 resulted in a significant decrease in the percentage of cells in the G1 phase, but an increased percentage of cells in the S phase (Figure 5B), suggesting that overexpression of miR-132 promotes progression of the cell cycle from the $\mathrm{G} 1$ to the $\mathrm{S}$ phase. Moreover, we analyzed the expression of $\mathrm{Ki}-67$, a marker of cellular proliferation (23), in keratinocytes with overexpressed or inhibited miR-132 (Figure 5C and Supplemental Figure 7A). In line with the results of the EdU incorporation assay, we found that Ki-67 expression was increased by the overexpression of miR-132 (1.9-fold change, $P=0.001$ ), but was decreased by the inhibition of endogenous miR-132 (0.81-fold change, $P=0.038)$. We next performed a colony formation assay to determine the effect of miR-132 on the long-term growth of keratinocytes. Overexpression of miR-132 increased (Figure 5, D and E), whereas inhibition of miR-132 suppressed the colony-forming capacity of human primary keratinocytes (Supplemental Figure 7, B and C). Taken together, these results demonstrate that miR-132 can regulate keratinocyte growth, which is reflected by both an immediate increase in cell cycle progression and long-term promotion of self-renewal.

To explore the mechanisms underlying the observed proproliferative effects of miR-132, we used specific chemical inhibitors, which target pathways known to regulate cell proliferation such as EGFR and its downstream signaling pathways, including the ERK, PI3K, PKC, and STAT pathways (24). Keratinocytes transfected with pre-miR-132 or control oligos were treated with SB203580 for p38 MAPK, SP600125 for JNK, U0126 for ERK, WP1066 for STAT3, Ro-31-8220 for PKC, wortmannin for PI3K, and PD153035 for EGFR (Figure 5F). Next, we analyzed the expression of Ki-67 by qRT-PCR. By comparing the inhibitor-treated cells with DMSO-treated cells (Figure 5F, black bars), we identified the pathways critical for cell proliferation, i.e., EGFR, JNK, ERK, STAT3, and PI3K, since Ki-67 expression was significantly reduced when these pathways were blocked.
Next, we compared miR-132-overexpressed cells with cells transfected with control oligos (Figure 5F, black versus white bars). miR-132 overexpression did not induce the same level of Ki-67 expression in the inhibitor-treated cells as it did in the DMSOtreated cells (1.9-fold). For example, miR-132 overexpression increased Ki- 67 by 1.1-fold with ERK inhibition, 1.4-fold with STAT3 inhibition, and 1.4-fold with EGFR inhibition, indicating that the function of miR-132 is compromised when these pathways are blocked and suggesting that the proproliferative effects of miR-132 may be mediated through these pathways.

To test whether miR-132 directly acts on EGFR, ERK, and STAT3 signals, we overexpressed miR-132 in keratinocytes and detected its effects on the phosphorylation of EGFR, ERK, and STAT3 in keratinocytes treated with EGF or TNF- $\alpha$, both of which reflect the activation status of these pathways. Both Western blotting (Figure 5G and Supplemental Figure 7D) and immunofluorescence staining (Figure $5 \mathrm{H}$ ) showed that overexpression of miR-132 increased the phosphorylation of EGFR, STAT3, and ERK, suggesting that miR-132 promotes keratinocyte proliferation by increasing the activity of these signals.

$H B-E G F$ is targeted by miR-132 in keratinocytes. Since miRs exert their biological functions by regulating their target genes, we aimed to identify the gene(s) directly targeted by miR-132 that may be responsible for the observed functional effects. Among the transcripts containing predicted miR-132-binding sites that were downregulated by miR-132 in keratinocytes (Supplemental Table 1), heparin-binding EGF-like growth factor (HB-EGF) occupies a top position. HB-EGF is one of the ligands that binds to and activates EGFR (25). The TargetScan algorithm identified 2 conserved binding sites for miR-132 in the 3'-UTR of $H B-E G F$ (Figure 6A). Moreover, $H B-E G F$ has been previously demonstrated as a direct target of miR-132 in human mast cells (26). Therefore, we set out to examine whether $H B-E G F$ is also targeted by miR-132 in human keratinocytes. We performed 3'-UTR luciferase reporter assays with luciferase reporter constructs containing the full-length $3^{\prime}$-UTR of HB-EGF mRNA in human primary keratinocytes (Figure 6B). We observed that WT HB-EGF 3'-UTR luciferase activity was decreased 3-fold $(P=0.0001)$ by pre-miR-132 compared with control precursor RNA (pre-miR-Ctrl). Mutation of either of the predicted target sites (Mut1 or Mut2) completely abolished the effect of miR-132 on reporter gene expression. These data demonstrate that miR132 directly targets $H B-E G F$ expression in human keratinocytes.

Furthermore, we analyzed the expression of HB-EGF in keratinocytes transfected with pre-miR-132 or anti-miR-132, followed by treatment with TNF- $\alpha$. qRT-PCR results showed that TNF- $\alpha$ induced the expression of $H B-E G F$ mRNA, while miR-132 suppressed it (Figure 6C). Like the other EGFR ligands, HB-EGF protein is first synthesized as a membrane-bound precursor that is further cleaved by metalloproteinase to produce the soluble mature form (25). Therefore, we performed Western blotting to analyze the amounts of total and cell surface HB-EGF protein in keratinocytes (Figure 6, D and E). Also, we performed ELISA to examine the mature form of HB-EGF secreted in the culture medium of keratinocytes (Figure $6 \mathrm{~F}$ ). These protein data further confirm that miR-132 downregulates HB-EGF expression in keratinocytes. Moreover, we performed qRT-PCR to analyze $H B-E G F$ 


\section{A}
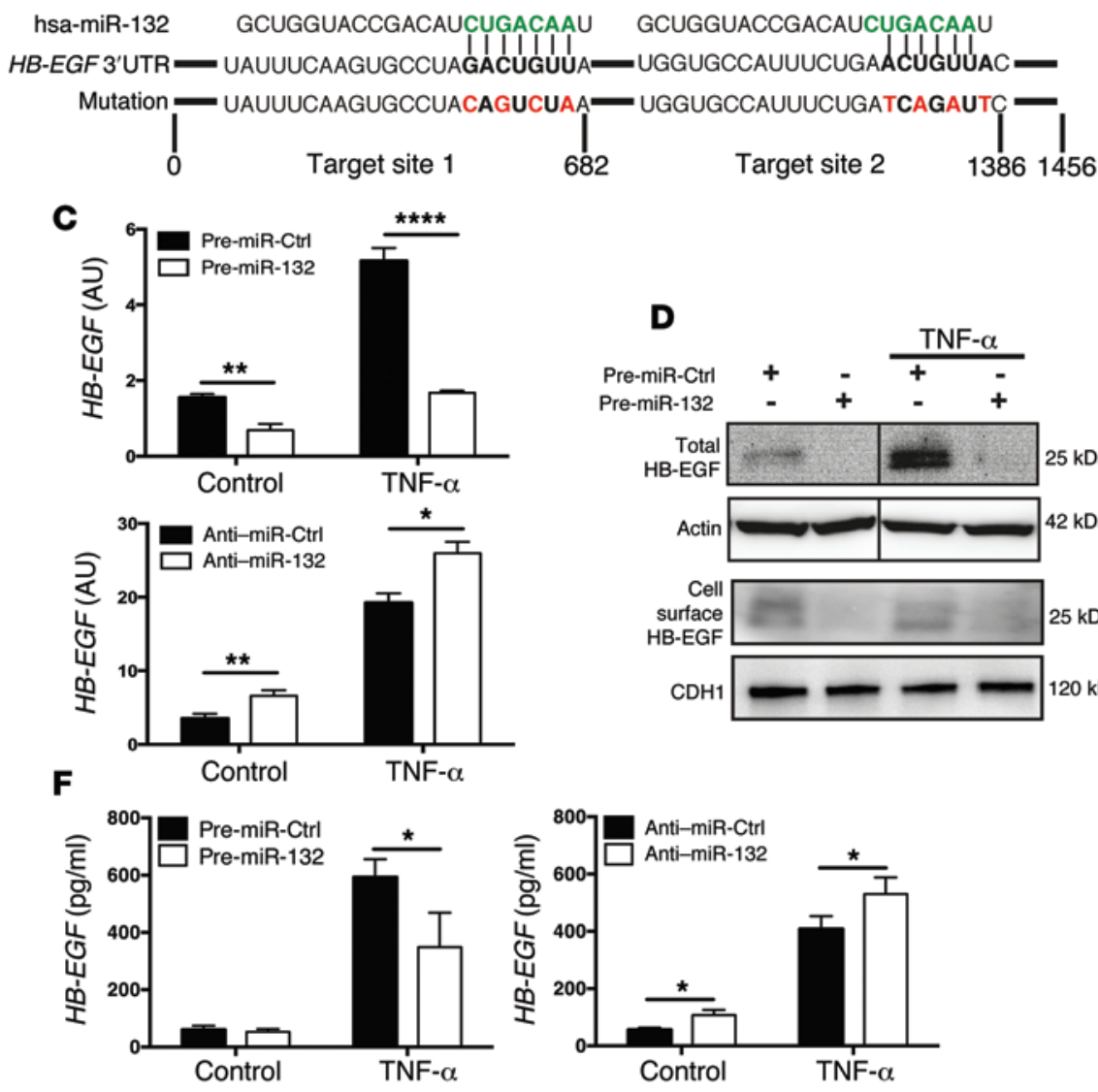
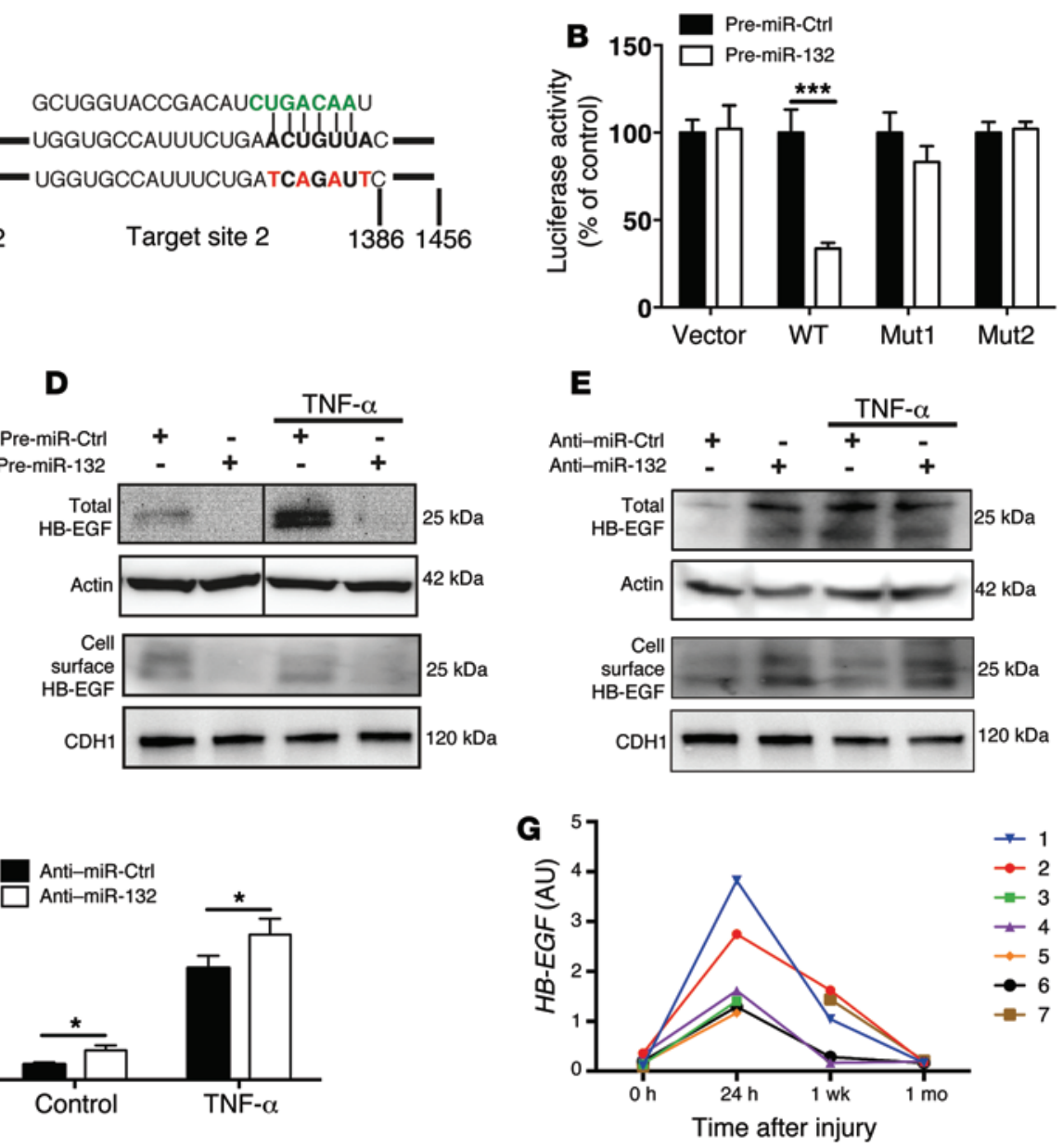

Figure 6. HB-EGF is targeted by miR-132 in keratinocytes. (A) Nucleotide resolution of the predicted miR-132-binding sites in the 3'-UTR of HB-EGF mRNA: seed sequence (green letters) and mutated miR-132-binding sites (red letters). (B) Keratinocytes were transfected with luciferase reporter plasmid containing WT or mutant (Mut) HB-EGF 3'-UTR or empty vector (Vector), together with pre-miR-132 or pre-miR-Ctrl. Luciferase activity was measured 24 hours later $(n=3)$. (C) qRT-PCR analysis of HB-EGF mRNA expression in keratinocytes transfected with pre-miR-132/pre-miR-Ctrl/anti-miR-132/anti-miR-Ctrl for 48 hours, then treated with TNF- $\alpha$ for 24 hours $(n=3)$. Total and cell surface HB-EGF protein were analyzed by Western blotting in cells transfected with pre-miR-132/pre-miR-Ctrl (D) or with anti-miR-132/anti-miR-Ctrl (E). (F) HB-EGF shed into culture medium was analyzed by ELISA ( $n=3)$. (C) qRT-PCR analysis of $H B$-EGF expression in wound biopsies from healthy donors $(n=7)$ at 0 hours, 24 hours, 1 week, and 1 month after injury. ${ }^{*} P<0.05,{ }^{* *} P<0.01$, ${ }^{* *} P<0.001$, and ${ }^{* * *} P<0.0001$ by Student's $t$ test.

expression in human wound biopsies collected from healthy donors at different time points after injury (Figure $6 \mathrm{G})$. We found that $H B-E G F$ was induced 24 hours after injury (inflammatory phase); however, its levels decreased after 1 week (proliferative phase) and reached the basal level 1 month (remodeling phase) after injury (Figure 6G). Given the expression pattern of miR-132 during the wound-healing process (Figure 2A), in which miR-132 expression levels increased at 24 hours and reached their peak 1 week after injury, we hypothesize that miR-132 may act as a feedback mechanism that dampens $H B-E G F$ expression in the proliferative phase of the skin wound-healing process.

To determine whether HB-EGF can mediate the observed biological effects of miR-132, we silenced $H B-E G F$ expression using a specific siRNA. An efficient decrease in HB-EGF expression levels was confirmed by qRT-PCR and Western blotting (Supplemental Figure 8, A and B). Silencing of $H B-E G F$ increased the expression of Ki-67 (Figure 7A) and the colony-forming ability of keratinocytes (Figure 7B), indicating that HB-EGF suppresses keratinocyte growth, in accordance with a previous study by Stoll et al. (27). In line with this, we found that silencing of $H B-E G F$ expression increased EGFR, STAT3, and ERK signaling, as shown by both Western blotting (Figure 7C and Supplemental Figure 8C) and immunofluorescence staining (Figure 7D).

Moreover, we analyzed the effects of HB-EGF on chemokine production by keratinocytes (Figure 7E) and found that $I L 8$, CCL2O, and CXCL5 mRNA levels were reduced by silencing $H B-E G F$. We also analyzed NF-אB pathway activity and found that silencing of $H B-E G F$, similar to overexpression of miR-132, suppressed NF- $\mathrm{kB}$ signal activation, which may explain the effects of $H B-E G F$ on chemokine production (Figure $7 \mathrm{~F}$ ).

Altogether, our results demonstrate that silencing of $H B-E G F$ can mimic the effects of miR-132 overexpression in keratinocytes, i.e., inhibit inflammation, promote cell growth, and regulate EGFR, STAT3, ERK, and NF- $\mathrm{KB}$ pathway activity. Therefore, these findings suggest that $H B-E G F$ is an important target for miR-132 function in keratinocytes. 


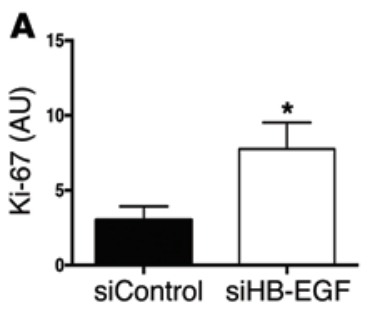

B
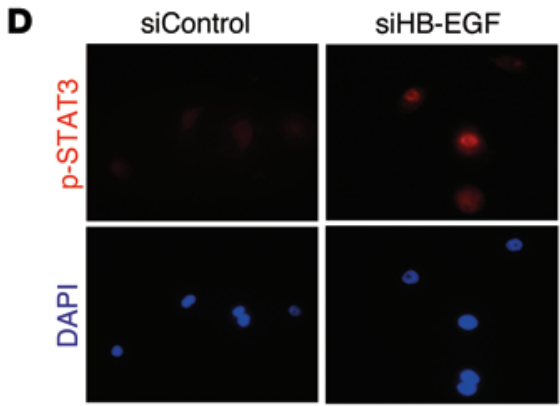

E

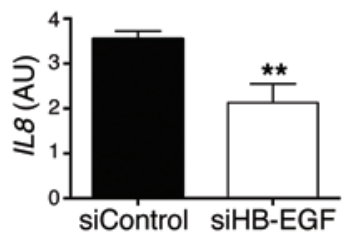

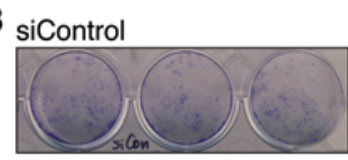

siHB-EGF

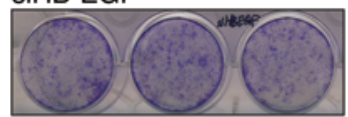

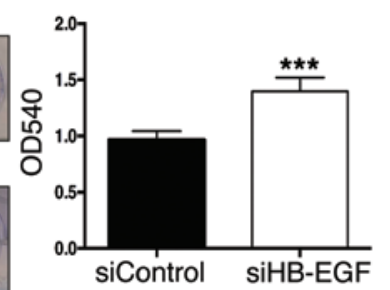

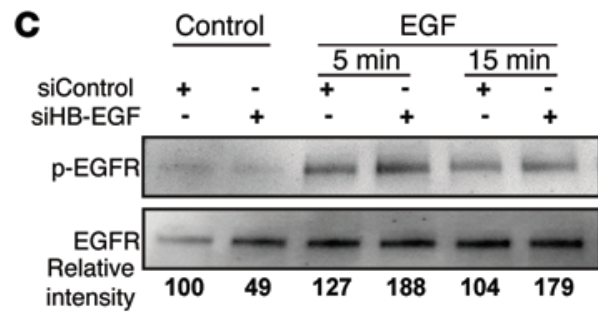

(p-EGFR/EGFR)

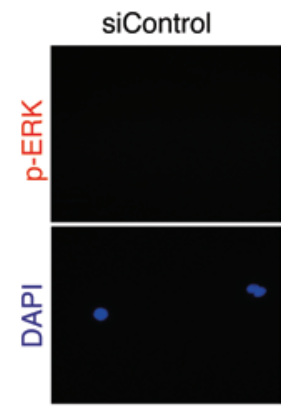

siHB-EGF
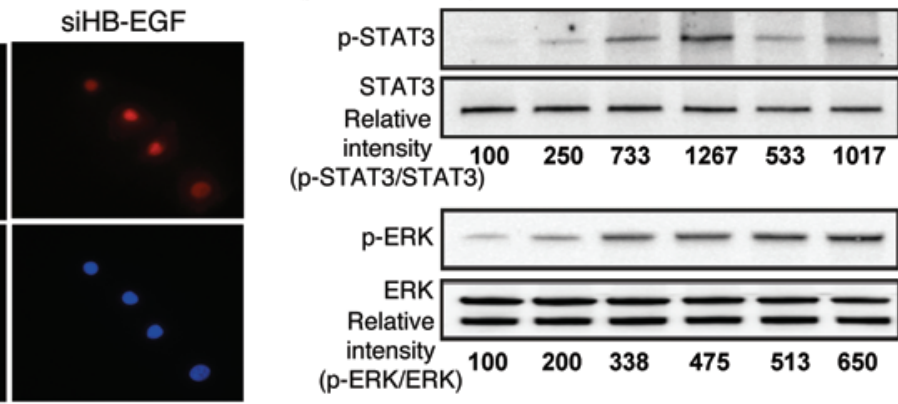

p-STAT3/STAT3)
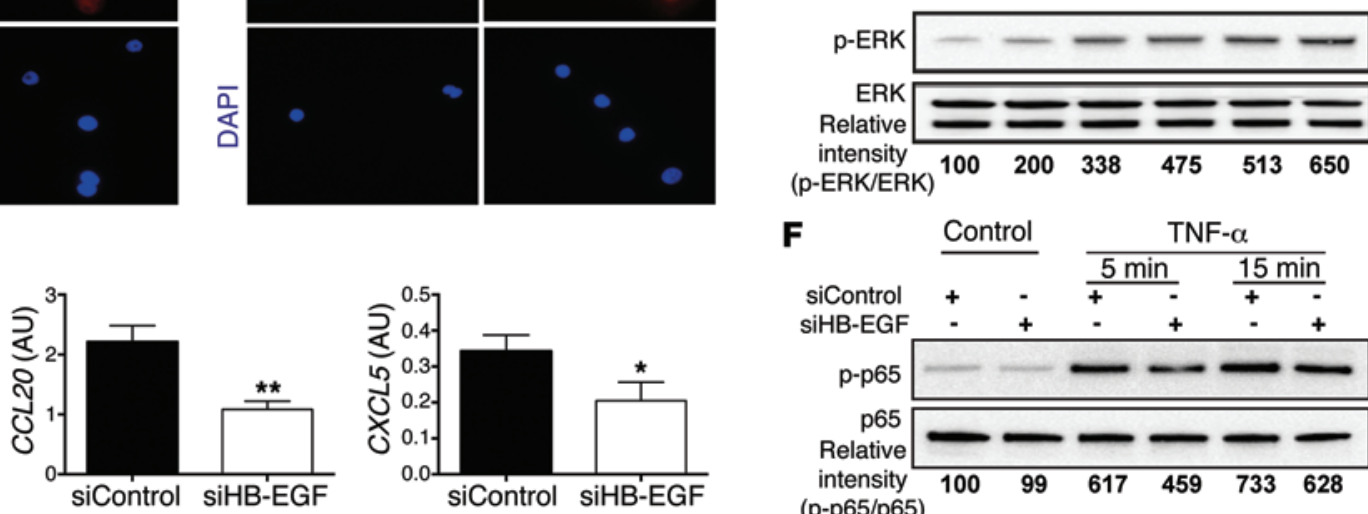

F

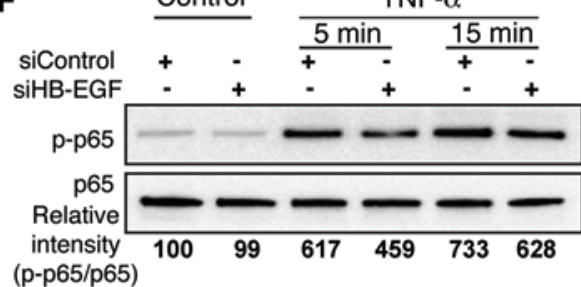

Figure 7. Silencing of HB-ECF can mimic the effects of miR-132 overexpression in keratinocytes. qRT-PCR analysis of Ki-67 expression $(n=3)(A)$ and colony formation assays $(n=6)(B)$ were performed in keratinocytes transfected with HB-EGF-specific siRNA (siHB-EGF) or control siRNA (siControl). (C) Both phosphorylated and total EGFR, STAT3, and ERK were detected by Western blot analysis in EGF-treated keratinocytes with silenced HB-EGF expression. (D) Immunostaining of p-STAT3 and p-ERK in keratinocytes transfected with siControl or siHB-EGF for 48 hours, then treated with EGF for 15 minutes. Cells were counterstained with DAPI (blue, nucleus). (E) qRT-PCR analysis of chemokine expression in keratinocytes transfected with siControl or siHB-EGF $(n=3)$. (F) Both phosphorylated and total p65 were detected by Western blotting in TNF- $\alpha$-treated keratinocytes with silenced HB-EGF expression. ${ }^{*} P<0.05,{ }^{* *} P<0.01$, and ${ }^{* *} P<0.001$ by Student's $t$ test.

Lack of miR-132 delays skin wound healing in vivo. To study the function of miR-132 in the skin in vivo, we used a mouse line containing a genetic deletion of the miR-132 locus (28). The lack of miR-132 expression in the skin of this mouse line was confirmed by qRT-PCR (Supplemental Figure 9). We collected skin biopsies from miR-132-KO mice $(n=9)$ and their WT littermates $(n=5)$ and performed histological analysis. We found that the epidermis of the KO mice was significantly $(P=0.007)$ thinner compared with that of the WT mice (Figure 8A). H\&E staining of the skin sections revealed that the epidermis of the WT mice was composed of 2 to 4 layers of keratinocytes, compared with 1 to 2 layers on average for the KO mice (Figure 8A). The thin epidermis phenotype in the KO mice reflects impaired proliferation of keratinocytes during skin development, which was confirmed by staining for the proliferation marker Ki-67, i.e., there were fewer Ki-67-positive cells present in the basal layer of the epidermis of the KO mice compared with that of the WT mice (Figure 8B). Consistently, we also observed increased expression of the miR-132 target HB-EGF in the epidermis of the KO mice in comparison with that of the WT mice (Figure 8B).

Furthermore, we isolated keratinocytes from the mouse skin and analyzed their biological behavior in vitro. Hbegf mRNA levels in keratinocytes from KO mice were increased compared with those in WT mice (Figure 8C). Keratinocytes from KO mice produced significantly higher amounts of Ccl20, Cxcl5, Tnf, $C x c l 1, I l 1 a$, and IL1b mRNA than did those from WT mice when treated with TNF- $\alpha$ (Figure $8 \mathrm{C}$ ). Consistently, by analyzing RNA from the mouse skin biopsies, we found that $C c l 2 O$ mRNA levels were significantly higher in the skin of KO mice compared with that of WT mice (Figure 9B).

Next, we compared the wound-healing process between KO and WT mice. We observed that the healing speed of KO mice $(n=9)$ was slower than that of their WT littermates $(n=6)$ $(P=0.04)$ (Figure 9A). We collected skin biopsies 2 days after injury, when the first difference in wound-healing rates was observed. We found that injury increased mRNA expression levels of Ccl2O, Cxcl5, Tnf, Cxcl1, Il1a, and Il1b and that there was a higher amount of these chemokines and cytokines in the wounds of KO mice $(n=9)$ compared with those of WT mice $(n=5)$ (Figure 9B). We further characterized the phenotypes of infiltrating leukocytes in the 2-day-old wounds by staining the specific immune cell markers, i.e., Gr-1 for neutrophils, CD68 for macrophages, and CD4 for Th cells. We found an increased number of neutrophils infiltrating into the wound beds of KO mice compared with WT mice (Figure 9C), even though their intrinsic ability to migrate toward chemoattractants was not affected by miR-132 KO (Figure 9D). This may be 
A
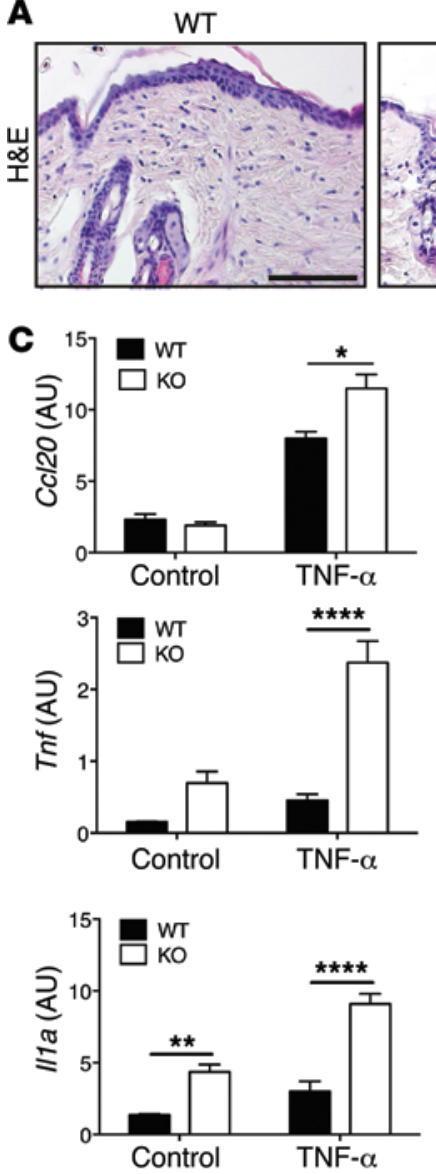

$\mathrm{KO}$
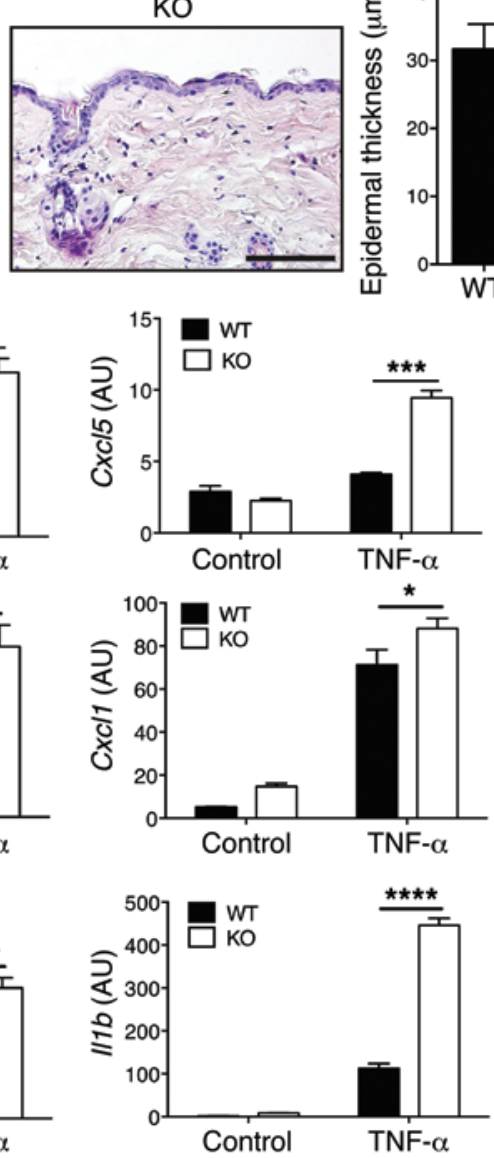

B
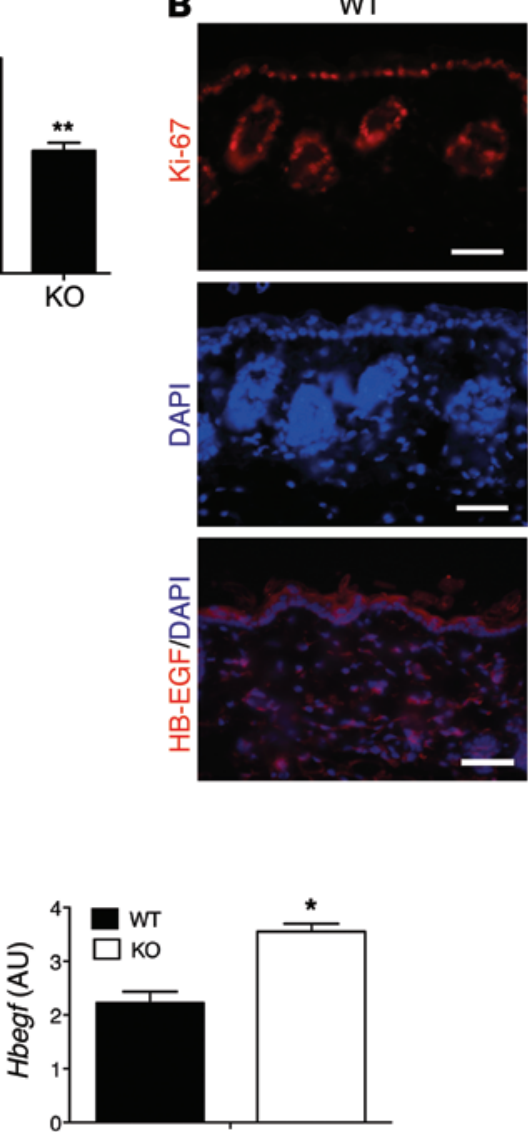
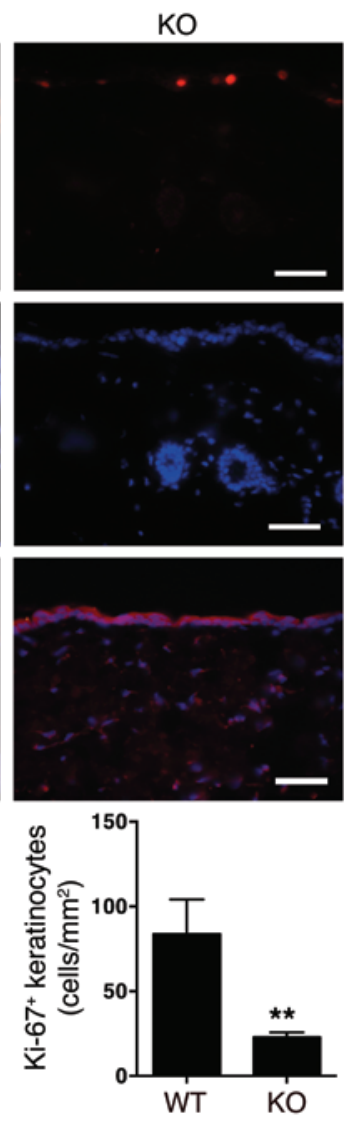

Figure 8. Decreased proliferation and increased chemokine production of epidermal keratinocytes from miR-132-KO mice. (A) H\&E staining of the skin from miR-132-KO mice $(n=9)$ and their WT littermate controls $(n=5)$ and epidermis thickness were analyzed. Scale bars: $100 \mu \mathrm{m}$. (B) Immunostaining of Ki-67 and HB-EGF in skin from WT $(n=9)$ and KO mice $(n=5)$. Sections were counterstained using DAPI (blue, nucleus). The number of Ki-67-positive cells was counted. Scale bars: $50 \mu \mathrm{m}$. (C) Epidermal keratinocytes were isolated from the skin of WT $(n=3)$ and KO ( $n=3) \mathrm{mice}$. CcI20, Cxcl5, Cxcl1, Tnf, I/1a, II1b, and Hbegf expression levels were analyzed using qRT-PCR. ${ }^{*} P<0.05,{ }^{*} P<0.01,{ }^{*}{ }^{*} P<0.001$, and ${ }^{* * *} P<0.0001$ by Student's $t$ test.

due to the increased expression by keratinocytes of the neutrophilattracting chemokines IL-8, CXCL1, and CXCL5 in the wounds of miR-132-KO mice (Figure 9B). In line with previous studies (29), we detected few Th cells and macrophages in the 2-day-old wounds and no obvious difference in these cell numbers between the KO and WT mice (data not shown).

Moreover, we observed a decreased number of Ki-67-positive cells and increased expression of Hbegf in the wound-edge epidermis of KO mice compared with that of WT mice (Figure 9E). We further stained the phosphorylated ERK (p-ERK) and STAT3 (p-STAT3) in the mice wounds, which showed less activation of these 2 pathways in the wound-edge epidermis of miR-132-KO mice compared with that of WT mice (Figure 9F). This is in line with our in vitro data (Figure 5, G and $\mathrm{H}$ ) and provides a mechanistic basis for the impaired proliferation observed in the wounds of miR-132-KO mice.

To further examine whether the increased expression of miR-132 in wounds is important for healing, we injected miR-132 inhibitors or control oligos intradermally into the wound edges of WT mice immediately and 4 days after the skin was injured (Figure 10A), which efficiently blocked increased miR-132 expression in the wounds (Figure 10B). In line with the data obtained from miR-132-KO mice, injection of miR-132 inhibitors significantly reduced Hbegf expression (Figure 10C), wound-healing speed (Figure 10D), and keratinocyte proliferation $\left(\mathrm{Ki}^{+} \mathrm{7}^{+}\right.$) at the wound edge (Figure 10E), while these inhibitors increased the expression of inflammatory cytokines such as Illb and Tnfin the wounds (Figure 10F). Moreover, we injected recombinant HB-EGF protein into the wound edges of WT mice (Supplemental Figure 10A), which also led to increased inflammatory cytokine expression (Supplemental Figure 10B), indicating that HB-EGF is an important target mediating the function of miR-132 in vivo. These results confirm the critical roles of miR-132 expression activation during skin wound repair.

During the wound-healing process in mouse skin, contraction of the dermis plays an important role. Herein, we transfected miR-132 inhibitors into mouse dermal fibroblasts, which did not change the contraction capacity of cells, indicating that miR-132 does not primarily affect the contraction of dermal fibroblasts during wound healing (Supplemental Figure 11).

Taken together, we conclude that lack of miR-132 expression results in delayed skin wound healing, accompanied by severe inflammation and deficient proliferation of keratinocytes. 
Inhibition of miR-132 delays reepithelialization of human ex vivo skin wounds. To study whether interference with miR-132 in a complex setting can affect human skin wound healing, we used a human ex vivo wound model. Full-thickness wounds were made on human skin obtained from plastic surgery, and the wounds were excised and transferred to cell culture plates containing medium. Such wounds reproducibly reepithelialize within 4 to 7 days (30). We treated these wounds topically with miR-132 inhibitors after injury, and the efficiency of miR-132 blocking was confirmed by qRT-PCR (Figure 11A). Histological analyses showed that inhibition of miR-132 delayed reepithelialization (Figure 11B) and reduced the number of $\mathrm{Ki}-67$-positive cells at the wound edges (Figure 11C). Moreover, we found that compared with the control wounds, miR-132 inhibitor-treated wounds expressed more inflammatory chemokines, e.g., CXCL1, CXCL5, and CCL20 (Figure 11D). Accordingly, they also had higher levels of HB-EGF (Figure 11E). Our data from a human ex vivo wound model further confirm that miR-132 is a critical player during human skin wound healing through its regulation of both keratinocyte proliferation and chemokine production by keratinocytes.

\section{Discussion}

In this study, we characterized the dynamic change of the miRNome during the inflammatory phase of human skin wound healing. We focused on one of the most highly upregulated miRs, miR-132, and studied its expression and function in wound healing. Our results suggest a model in which TGF- $\beta$ induces miR-132 expression in wound-edge keratinocytes. Through the regulation of HB-EGF and other targets, miR-132, on the one hand, decreases NF-kB activity and restricts inflammation and, on the other, increases the activity of STAT3 and ERK, thus promoting cell growth (Figure 11F). We conclude that miR-132 may act as a critical regulator facilitating the transition from the inflammatory to the proliferative phase during skin wound healing.

By screening the effects of wound healing-related factors on miR-132 expression in keratinocytes, we found that TGF- $\beta 1$ and TGF- $\beta 2$ induced miR-132 expression. TGF- $\beta$ s are pluripotent cytokines that maintain skin homeostasis, and both TGF- $\beta 1$ and TGF- $\beta 2$ are upregulated in wounds, where they promote keratinocyte migration during reepithelialization (reviewed in ref. 12). It has been shown that TGF- $\beta$ inhibits epidermal keratinocyte proliferation (12), whereas here we found that miR-132, which is induced by TGF- $\beta$, actually increased keratinocyte proliferation. We hypothesize that induction of miR-132 expression may be a novel way for TGF- $\beta$ to reduce its negative effect on keratinocyte growth during wound repair, and this will be interesting to test in a future study. Binding of TGF- $\beta$ to its receptors T $\beta$ R-II and T $\beta R$-I leads to the activation of downstream Smad proteins, which induce the expression of TGF- $\beta$ target genes (reviewed in ref. 12). Recently, it was shown in pancreatic cancer cells that transcription factor Sp1 plays a major role in MIR132 gene expression (31). In the same cell type, it has been known that TGF- $\beta$ stimulates formation of the Smad-Sp1 complex, resulting in super induction of Sp1mediated transcription (32). Since the interaction between Smad and $\mathrm{Sp} 1$ is dependent on both cell type and cell-activation state (32), further study is needed to determine whether Sp1 mediates the induction effects of TGF- $\beta$ on miR-132 in keratinocytes.
Restraining inflammation to an appropriate level is critical for skin wound healing, since excessive and persistent inflammation results in failure to enter the proliferative phase and impairs healing $(1,2)$. Here, we show that in human primary keratinocytes, miR-132 suppresses the production of multiple chemokines, such as IL-8, CXCL5, CXCL1, and CCL20, and reduces the capability of keratinocytes to activate endothelial cells and attract neutrophils. The continuous increase in miR-132 expression levels during the inflammatory and proliferative phases of wound healing may contribute to limiting the severity of inflammation and facilitating normal reepithelialization. In line with this, it has been reported that in several types of immune cells (e.g., monocytes, macrophages, and mast cells) and lymphatic endothelial cells, miR-132 expression is induced by different proinflammatory molecules and functions as a negative feedback mechanism to resolve inflammation (reviewed in ref. 33). Furthermore, we show that the antiinflammatory effect of miR-132 is mediated through $\mathrm{NF}-\kappa \mathrm{Bs}$, which are ubiquitous transcription factors held in the cytoplasm in an inactive state by specific inhibitors in resting cells. Upon degradation of the inhibitor, for example in response to certain cytokines such as TNF- $\alpha$ or IL-1 $\beta$, NF- $\kappa$ B translocates to the nucleus and activates the transcription of inflammatory genes (reviewed in ref. 22). p65 is one of the essential transcription factors among the NF- $\mathrm{B}$ family members, and its nuclear translocation and phosphorylation represent the activation of $\mathrm{NF}-\kappa \mathrm{B}$ signaling (22). Here, we show that miR-132 suppresses NF- $\mathrm{B}$ pathway activity by measuring its effects on NF- $\kappa \mathrm{B}$-dependent luciferase reporter gene expression, the amount of p65 entering cell nuclei, and the level of phosphorylated p65.

Moreover, we found that miR-132 promoted the growth of keratinocytes. A number of previous reports have suggested that miR-132 can regulate cell proliferation, especially in cancer. For example, in osteosarcoma (34), ductal carcinoma in situ (35), and pancreatic cancer (31), miR-132 inhibits cancer cell proliferation. However, in human hemangiomas, miR-132 increases endothelial cell proliferation and tube-forming capacity (36). Here, we show that in human keratinocytes, miR-132 promotes cell cycle progression and long-term self-renewal. Transcriptomic analysis revealed that many genes critical for cell proliferation were upregulated by miR-132 in keratinocytes. Moreover, we identified STAT3 and ERK pathways critical for the progrowth effect of miR-132 in keratinocytes. Both STAT3 and ERK convey signals to the nucleus upon stimulation with cytokines and growth factors, leading to transcriptional activation of the downstream genes important for cell proliferation, migration, survival, and oncogenesis $(37,38)$. Activation of STAT3 has been shown to promote skin wound healing and tumor development (37). Here, we show that miR-132 increases the activity of both STAT3 and ERK pathways, thus contributing to the proliferation of keratinocytes.

In this study, we focused on one target of miR-132- HB-EGF - which is one of the EGFR ligands, in addition to TGF $\alpha$, amphiregulin (AR), epiregulin (EREG), and $\beta$ cellulin, that is expressed by keratinocytes in an autocrine fashion (39, 40). Unlike the other EGFs, overexpression of HB-EGF in keratinocytes was previously reported to lead to a large decrease in cell 

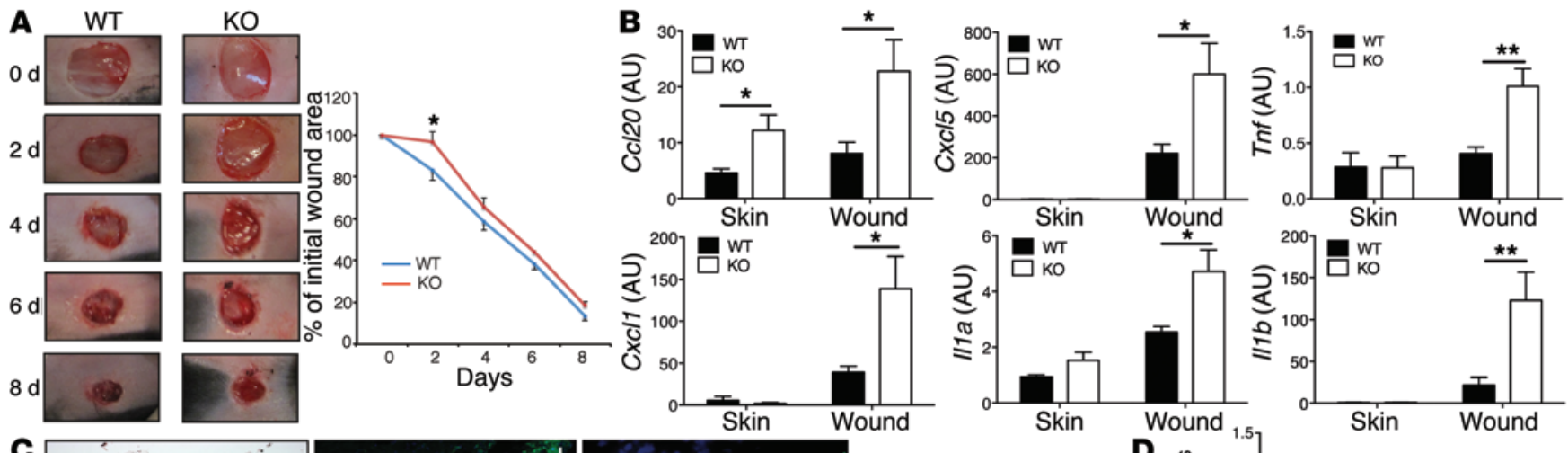

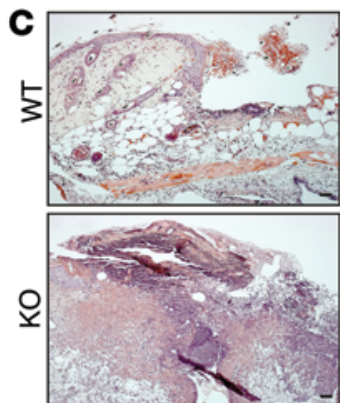

$\mathrm{H} \& \mathrm{E}$

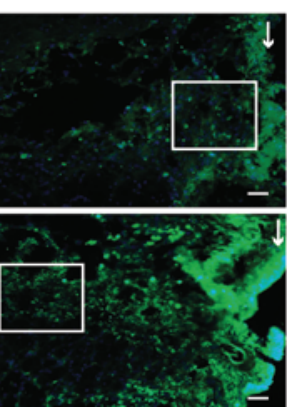

Gr-1/DAPI

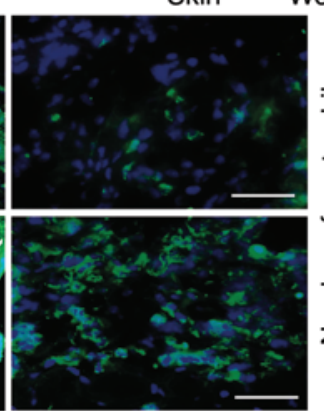

Gr-1/DAPI
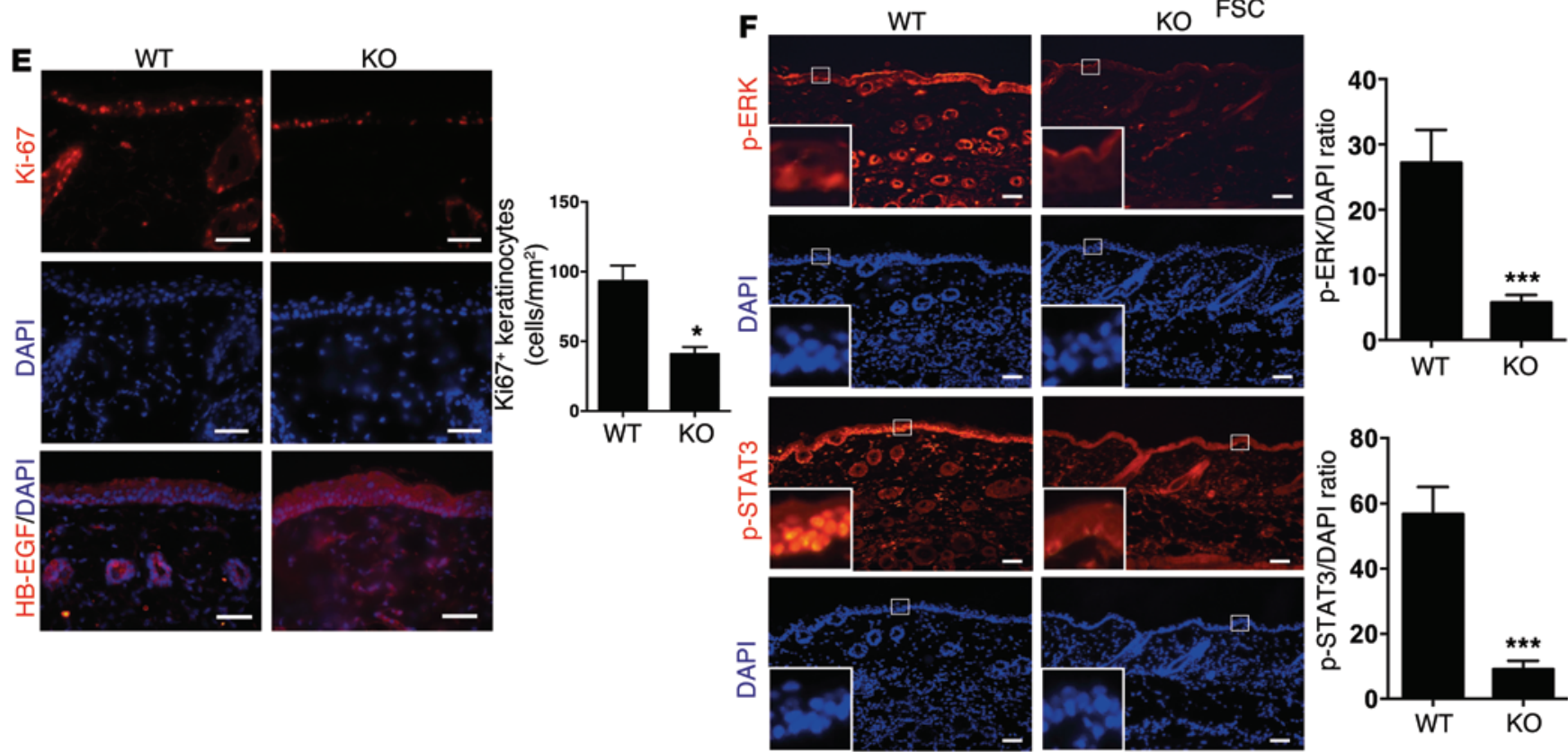

Figure 9. Skin wound healing is impaired in miR-132-KO mice. (A) Representative images of wounds on the backs of miR-132-KO mice $(n=9)$ and their WT littermate controls $(n=6)$ on days $0,2,4,6$, and 8 after wounding. Wound closures were quantified and are presented as the percentage of the initial wound area size. (B) qRT-PCR was performed to detect $C \mathrm{C} / 20, C x \mathrm{C} / 5$, Cxc/1, Tnf, I/1a, and I/1b expression levels in the intact and wound-edge skin of both WT $(n=5)$ and KO $(n=9)$ mice 2 days after injury. ${ }^{*} P<0.05$ and ${ }^{* *} P<0.01$ by Student's $t$ test. (C) H\&E staining (left panels) and immunostaining of Gr-1 (middle and right panels) in the wound area in WT and KO mice 2 days after injury. White arrows demarcate wound edges. Scale bars: $50 \mu \mathrm{m}$. (D) Chemotaxis of neutrophils isolated from the peripheral blood of WT $(n=5)$ and KO mice $(n=7)$ toward the medium containing $8 \mu \mathrm{M} \mathrm{N}$-formylmethionyl-leucyl-phenylaIanine (fMLP). Migrating cells were quantified by flow cytometry. Plots show the FSC/SSC of the migrated cells. (E) Immunostaining of Ki-67 and HB-EGF in wound-edge skin from WT $(n=9)$ and KO mice $(n=5) 2$ days after injury. Sections were counterstained using DAPI (blue, nucleus). The number of Ki-67positive cells was counted. (F) Immunostaining of p-STAT3 and p-ERK in wound-edge skin from WT $(n=9)$ and KO mice $(n=5) 2$ days after injury. Scale bars: $50 \mu \mathrm{m}$ (E and $\mathbf{F}) .{ }^{*} P<0.05,{ }^{* *} P<0.01$, and ${ }^{* * *} P<0.001$ by Student's $t$ test. 

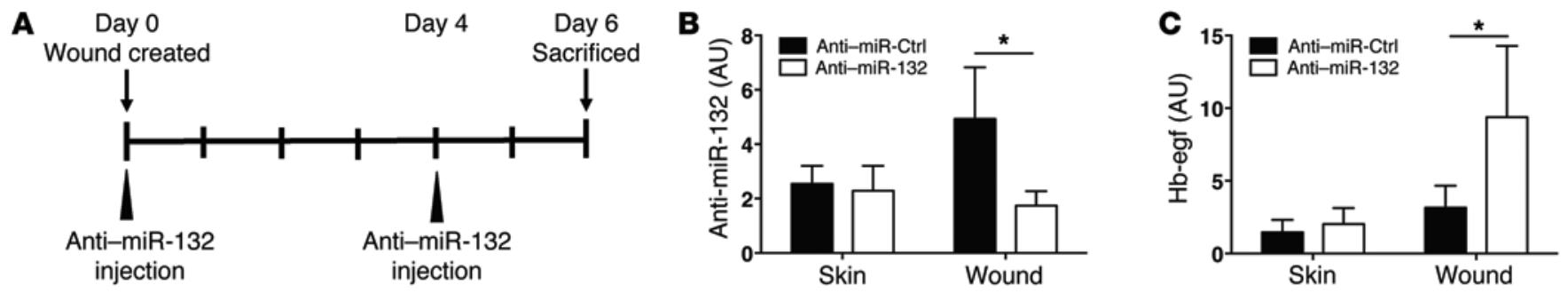

\section{D}
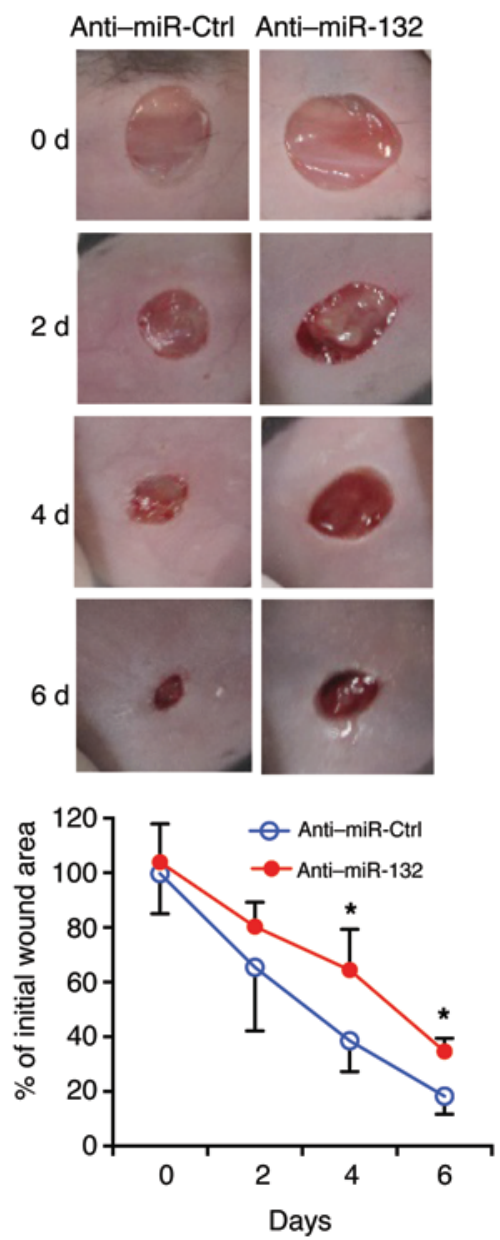

$\mathbf{E}$
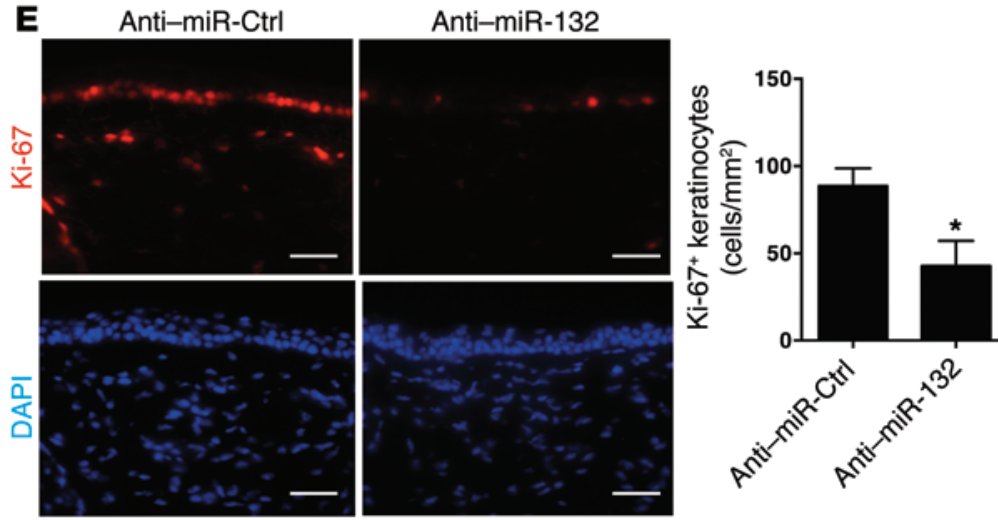

$\mathbf{F}$
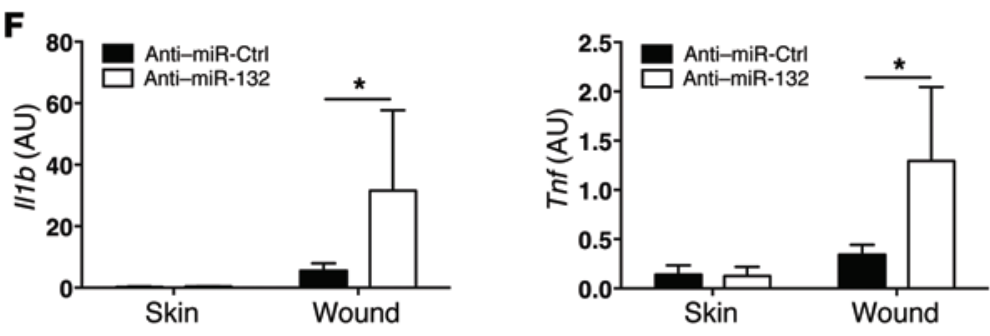

Figure 10. Inhibition of miR-132 in WT mouse wounds delays wound healing. (A) Anti-miR-132 or anti-miR-Ctrl was injected intradermally into the wound edges in mice ( $n=5$ for each group) immediately and 4 days after wounding. Skin biopsies at the wound site and intact area were collected 6 days after injury. miR-132 (B) and Hbegf (C) expression levels were analyzed using qRTPCR. (D) Representative images of wounds on days 0, 2, 4, and 6 after wounding. Wound closures were quantified and are presented as the percentage of the initial wound area size. (E) Immunostaining for Ki-67 in the wound-edge skin 6 days after injury. Sections were counterstained using DAPI. The number of Ki-67-positive cells was counted. Scale bars: $50 \mu \mathrm{m}$. (F) Expression of //1b and Tnf was detected in the intact and wound-edge skin of mice treated with anti-miR-Ctrl $(n=5)$ or anti-miR-132 $(n=5) 6$ days after injury using qRT-PCR. ${ }^{*} P<0.05$ by Student's $t$ test.

growth (27), and our results are in line with this finding. This may be caused by the fact that HB-EGF stimulation sorts the internalized EGFR for degradation, whereas TGF $\alpha, A R, E R E G$, and EGF stimulation leads to EGFR recycling $(27,41)$. In our study, we decreased the endogenous expression of HB-EGF in keratinocytes using siRNA, which may reduce EGFR occupancy and/or degradation by HB-EGF. Thus, adding extracellular EGF may induce stronger activation of EGFR and its downstream STAT3 and ERK signaling. Moreover, we found that HB-EGF silencing decreased NF- $\mathrm{KB}$ signaling activity and the expression of chemokines, which mimic the effects of miR-132 overexpression in keratinocytes, whereas injection of HB-EGF increased chemokine levels in the wounds in vivo. Notably, we show that the expression of HB-EGF is increased in human skin wounds in accordance with previous results (42). Because of the antiproliferative and proinflammatory effects of HB-EGF, it may be crit- ical to control the level of $\mathrm{HB}-\mathrm{EGF}$ in each phase of the repair process. Given the difference between miR-132 and HB-EGF expression patterns during wound healing, we hypothesize that the increase in miR-132 expression may be responsible for the decrease in HB-EGF expression during the proliferative compared with the inflammatory phase. Given its capacity to restrain HB-EGF expression, miR-132 may have a role in assuring the proper proliferation of keratinocytes during wound healing.

Next, we validated the importance of miR-132 for skin wound healing in vivo by using a miR-132-KO mouse line, which has been previously characterized with regard to its resistance to pressure overload-induced heart failure (28) and experimental autoimmune encephalomyelitis (43). No skin phenotype has been described so far. Here, we show that miR-132-KO mice have a thinner epidermis with fewer proliferating cells compared with that of their WT littermates. Upon injury, we observed 
A

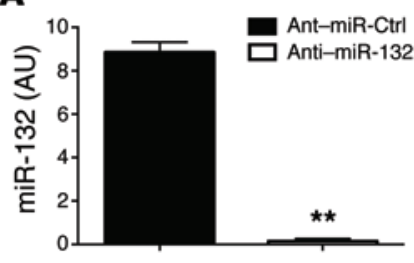

B

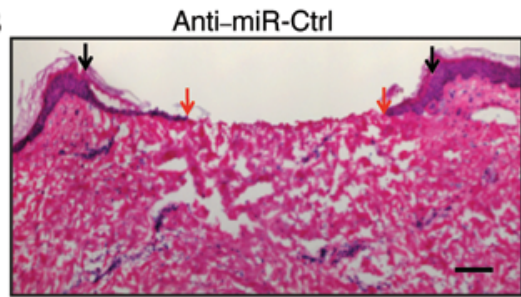

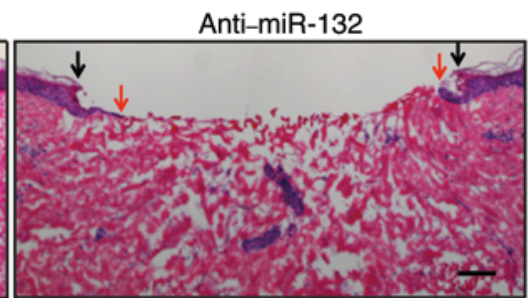

$\mathbf{E}$

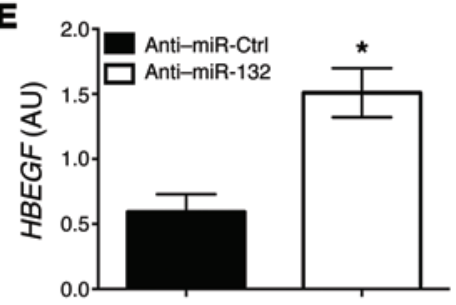

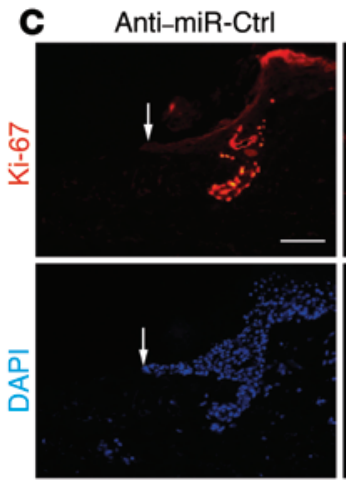
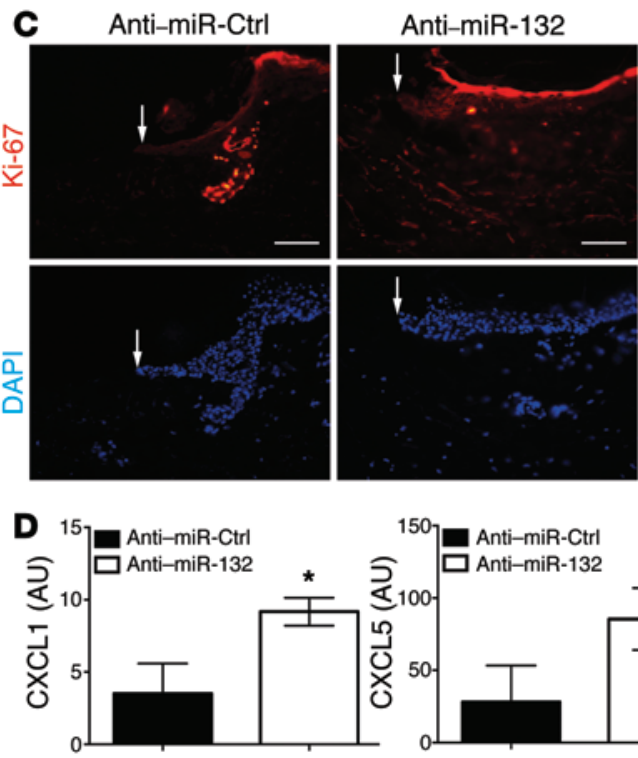

${ }^{1.5} \square$ Anti-miR-Ctrl

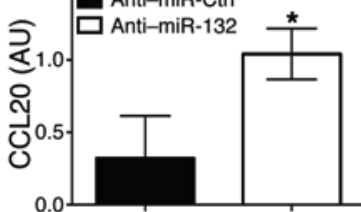

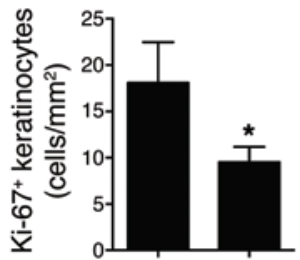
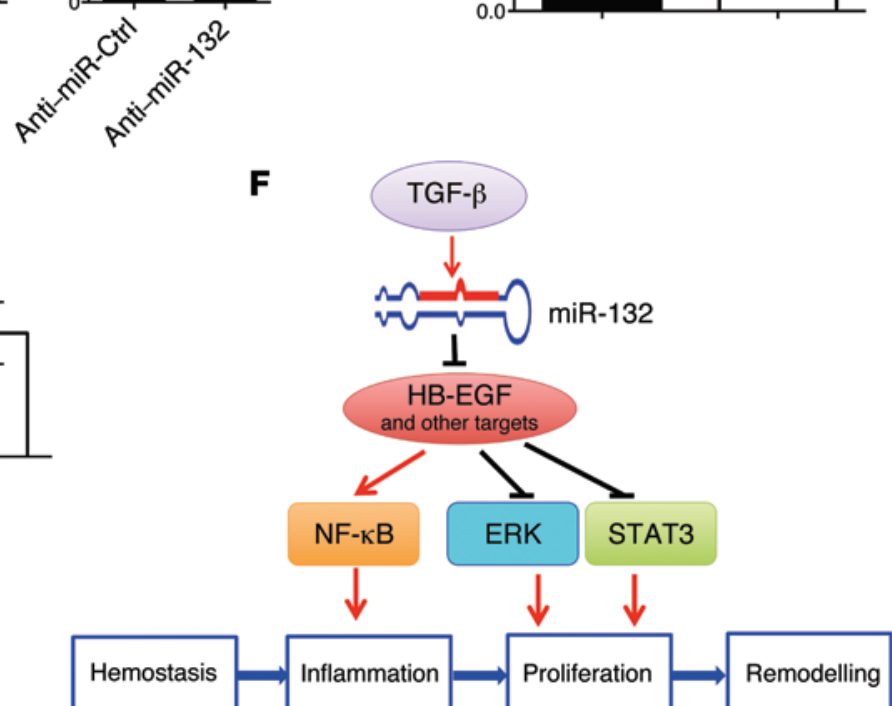

Figure 11. Inhibition of miR-132 delays reepithelialization of human ex vivo skin wounds. Human ex vivo skin wounds were treated topically with anti-miR-132 $(n=4)$ or anti-miR-Ctrl $(n=4)$ after injury, and miR-132 blocking efficiency was confirmed by qRT-PCR (A). (B) H\&E staining of the ex vivo skin wounds 3 days after injury. Black arrows demarcate the initial wound edges, while the red arrows indicate a newly formed epidermis. Scale bars: $200 \mu \mathrm{m}$. (C) Immunostaining of Ki-67 in ex vivo skin wounds 3 days after injury. Sections were counterstained with DAPI. The number of Ki-67positive cells was counted. White arrows demarcate the wound edges. Scale bars: $50 \mu \mathrm{m}$. CXCL1, CXCL5, CCL20 (D), and HB-EGF (E) expression levels were detected by qRT-PCR in ex vivo wounds treated with anti-miR-Ctrl $(n=4)$ or anti-miR-132 $(n=4) 3$ days after injury. (F) Schematic summary of the regulation and function of miR-132 during skin wound healing. ${ }^{*} P<0.05$ and ${ }^{*} P<0.01$ by Student's $t$ test.

higher chemokine and cytokine expression levels, more neutrophil infiltration, and decreased proliferation of epidermal keratinocytes in the wounds of KO mice compared with those of WT mice, which may explain the delayed wound-healing phenotype of miR-132-KO mice. Moreover, we injected miR-132 inhibitors into the wound edges of WT mice, which also delayed wound healing, similar to what we observed in miR-132-KO mice. These data confirm that activation of miR-132 expression is important for the normal wound-healing process. Finally, we validated these in vivo data from mouse wound models in human ex vivo skin wounds. Together, we conclude that miR-132 is an essential regulator of normal skin wound healing.

In summary, we have identified miR-132 as a novel regulator of cell proliferation and inflammatory chemokine and cytokine production in keratinocytes. Upregulation of miR-132 in keratinocytes during wound healing may be critical for facilitating the transition from the inflammatory to the proliferative phase. In chronic, nonhealing wounds, TGF- $\beta$ signaling, which is an important inducer of miR-132 expression, is attenuated (12). This may result in the deregulation of miR-132 expression and in turn contribute to the characteristic phenotype of persistent inflammation and the failure to enter the normal proliferative phase $(1,2)$. It would be important to further explore the expression and function of miR-132 in chronic wounds, since miR-132 may be an attractive therapeutic target due to its capacity to alleviate inflammation and promote keratinocyte proliferation.

\section{Methods}

Additional details are available in the Supplemental Methods.

Tissue samples. To study human skin wound healing in vivo, 14 healthy volunteers were enrolled at the Karolinska University Hospital Dermatology Clinics. Two 3-mm surgical wounds (wounds 1 
and 2) were created at the same time on the abdominal skin of each healthy volunteer (Supplemental Figure 1A). Twenty-four hours later, the edge from wound 1 was excised with a 6-mm biopsy punch and snap-frozen; 7 days after injury, the edge from wound 2 was collected; 31 days after wounding, the edge was excised from wound 1 again. The wound area was covered with sterile surgical film (Tegaderm; 3M Health Care).

RNA extraction and microRNA TLDA. Total RNA was extracted from tissues and cells using the miRNeasy Mini kit (QIAGEN). Skin biopsies from healthy volunteers and mice were homogenized in liquid nitrogen using a Mikro-Dismembrator U (B. Braun Biotech Inc.) prior to RNA extraction. The miRs were reverse transcribed and amplified using the multiplex RT TaqMan MicroRNA Low Density Array (Life Technologies). Further details on TLDA are described in the Supplemental Methods.

qRT-PCR. Quantification of miRs by qRT-PCR was performed as previously described (44). miR expression levels were normalized between different samples on the basis of the values of RNU48 RNA in humans and U6 snRNA in mice. The primary miR transcripts were quantified using the TaqMan Pri-miRNA assay (Hs03303111_pri; Life Technologies). In order to quantify mRNAs, total RNA was reverse transcribed using the RevertAid First Strand cDNA Synthesis Kit (Thermo Scientific Fermentas, Life Technologies). CCL2O, CXCL5, CXCL1, IL8, IL1A, IL1B, TNF, MKI67, HB-EGF, TGFB1, TGFB2, SELE, and VCAM1 mRNAs were quantified by TaqMan gene expression assays (Life Technologies). Target gene expression levels were normalized between samples to the internal control 18S rRNA (forward: 5'-CGGCTACCACATCCAAGGAA-3'; reverse: 5'-GCTGGAATTACCGCGGCT-3'; probe: 5'- FAM-TGCTGGCACCAGACTTGCCCTC-TAMRA-3') in humans and to Gapdh (Life Technologies) in mice.

$I S H$. ISH was performed on frozen sections (10 $\mu \mathrm{m}$ in thickness) of skin biopsies as previously described (44). Additional ISH details are provided in the Supplemental Methods.

Cell culture and treatments. Human adult epidermal keratinocytes were purchased from Cascade Biologics and cultured in EpiLife serum-free keratinocyte growth medium including Human Keratinocyte Growth Supplement at a final $\mathrm{Ca}^{2+}$ concentration of $0.06 \mathrm{mM}$ (Cascade Biologics, Life Technologies) at $37^{\circ} \mathrm{C}$ in $5 \% \mathrm{CO}_{2}$.

To study the biological effects of miR-132 on keratinocytes, third-passage keratinocytes at $30 \%$ to $50 \%$ confluence were transfected with $20 \mathrm{nM}$ pre-miR-132 or miR precursor negative control number 1 (pre-miR-Ctrl) (Ambion); 20 nM miR-132 miRCURY LNA Power inhibitor (anti-miR-132) or negative control A (anti-miR-Ctrl); and $30 \mathrm{nM}$ Silencer select predesigned siRNA for HB-EGF (siRNA ID: s4353) or siRNA negative control number 1 (Ambion) using Lipofectamine 2000 (Invitrogen).

Details on the cytokine and signaling pathway inhibitor treatments are described in the Supplemental Methods.

Protein detection. Cell culture supernatant from keratinocytes was collected, and protein levels of IL-8 (BioLegend), CXCL5 (R\&D Systems), CCL20 (Boster Immunoleader), and HB-EGF (Abcam) were measured by ELISA according to the manufacturer's instructions.

Immunofluorescence staining was performed on cultured human keratinocytes with the following antibodies: anti-human p65 (1:200; catalog 6956; Cell Signaling Technology); anti-human p-STAT3 (1:200; catalog 9131; Cell Signaling Technology); and anti-human
p-ERK (1:200; catalog 9101; Cell Signaling Technology). Primary antibodies were detected with Alexa Fluor 564-conjugated goat anti-rabbit IgG $(\mathrm{H}+\mathrm{L})$ or rabbit anti-goat IgG $(\mathrm{H}+\mathrm{L})$ (catalogs A11071 and A21085, respectively; Life Technologies) and visualized with a fluorescence microscope (Zeiss).

Keratinocyte whole-cell lysates extracted using radioimmunoprecipitation assay (RIPA) buffer, nuclear lysates extracted using a Nuclear Extract Kit (Active Motif), and cell membrane lysates extracted using a Mem-PER Plus Membrane Protein Extraction Kit (Thermo Scientific, Life Technologies) were analyzed by Western blotting with the following antibodies: anti-human p-p65 (1:1,000; catalog 3033; Cell Signaling Technology); anti-human p65 (1:1,000; catalog 6956; Cell Signaling Technology); anti-human p-STAT3 (1:1,000; catalog 9131; Cell Signaling Technology); anti-human STAT3 (1:1,000; catalog sc-8019; Santa Cruz Biotechnology Inc.); anti-human p-ERK (1:2,000; catalog 9101; Cell Signaling Technology); anti-human ERK (1:1,000; catalog 06-182; EMD Millipore); anti-human p-EGFR (1:1,000; catalog 2231; Cell Signaling Technology); anti-human EGFR (1:1,000; catalog 2239; Cell Signaling Technology); anti-human poly (ADP-ribose) polymerase (PARP) (1:2,000; catalog 556362; BD Biosciences); anti-human HB-EGF (1:1,000; catalog AF-259-NA; R\&D Systems); anti-human E-cadherin (CDH1) (1:1,000; catalog 610182; BD Biosciences); anti-human $\mathrm{p}-\mathrm{I} \kappa \mathrm{B} \alpha$ (1:1,000; catalog 2859; Cell Signaling Technology); and anti-human IкB $\alpha$ (1:1,000; catalog 4812; Cell Signaling Technology). Actin expression was visualized using HRP-coupled anti-human actin antibody (1:20,000; catalog A3854; Sigma-Aldrich).

Leukocyte chemotaxis assay. Additional details on the leukocyte chemotaxis assay are provided in the Supplemental Methods.

Cell cycle analysis. A Click-iT EdU flow cytometric assay (Invitrogen) was performed according to the manufacturer's instructions and analyzed on a FACScan flow cytometer (Beckman Coulter).

Colony formation assay. Cells were seeded into 12-well plates, with a density of 8,000 cells per well 24 hours after transfection. The medium was changed every third day. After 8 days, the colonies were stained with crystal violet (Sigma-Aldrich) and photographed. Methanol was used to dissolve crystal violet, and the absorbance value was measured at $540 \mathrm{~nm}$.

Gene expression microarray. Expression profiling of primary human keratinocytes transfected with $10 \mathrm{nM}$ pre-miR-132 or premiR-Ctrl for 48 hours (in triplicate) was performed using the Affymetrix GeneChip system at the microarray core facility of KI. Further details on the microarray analysis are provided in the Supplemental Methods. The data discussed herein have been deposited in the NCBI's Gene Expression Omnibus (GEO) (45) (GEO GSE61139).

Luciferase reporter assays. Renilla luciferase reporter plasmids containing synthetic sequence repeats that are fully complementary to miR-132 (miR-132 sensor) or to the 3'-UTR of the HB-EGF gene cloned downstream of the reporter gene and empty luciferase vector were obtained from SwitchGear Genomics. The mutations were generated with the predicted target sites of miR-132 on the $H B-E G F$ 3'-UTR using the QuickChange II XL Site-Directed Mutagenesis Kit (Stratagene, Agilent Technologies), according to the manufacturer's instructions. The NF- $\mathrm{KB}$ reporter plasmid pGL4.32 vector (luc2P/ NF- $\kappa$ B-RE/Hygro) was obtained from Promega and contains 5 copies of an NF-кB response element that drives transcription of the luciferase reporter gene luc2P (Photinus pyralis). Human primary keratino- 
cytes were cotransfected with the luciferase reporters $(250 \mathrm{ng} / \mathrm{ml})$, together with $10 \mathrm{nM}$ pre-miR-132 or pre-miR-Ctrl using the FuGENE HD transfection reagent (Promega). Luciferase activity was analyzed 24 hours after transfection using the LightSwitch Luciferase Assay reagent (SwitchGear Genomics) or the Dual-Luciferase Reporter Assay System (Promega).

In vivo wound models. C57BL/6N mice were caged individually for 1 week, handled daily, and then wounded. Details on the wounding process are described in the Supplemental Methods. TGF- $\beta$ R inhibitor SB431542 (10 nmol; Tocris) or DMSO (Figure 2, J and K), or HB-EGF protein ( $1 \mu \mathrm{g}$; R\&D Systems), or PBS (Supplemental Figure 11) was injected intradermally into the wound edges in the mice immediately after wounding, and skin biopsies at the wound site and intact area were collected 2 days later. In the experiment shown in Figure 10, $1 \mathrm{nmol}$ in vivo LNA miR-132 inhibitor (anti-miR-132) or negative control (anti-miR-Ctrl) (Exiqon) were injected intradermally into the wound edges in the mice immediately and 4 days after wounding. Skin biopsies at the wound site and intact area were collected 6 days after injury.

The C57BL/6N mouse line lacking miR-132 expression (KO) was generated previously (28). In the first experiment (9 KO versus $6 \mathrm{WT}$ mice), the size of the wound area was photographed and measured every other day until approximately $90 \%$ of the wound area was healed, as previously described (46). In the second experiment (9 KO vs. 5 WT mice), the mice were sacrificed the second day after injury, and skin biopsies at the wound site and intact area were collected.

The skin biopsies from a K5.TGF- $\beta 1$ Tg mouse were a gift of Gangwen Han and Xiao-Jing Wang (University of Colorado, Denver, Colorado, USA) $(13,14)$.

Histological analysis. Paraffin-embedded tissue sections $(5 \mu \mathrm{m}$ in thickness) were deparaffinized and rehydrated, followed by $\mathrm{H} \& \mathrm{E}$ staining. To evaluate the thickness of the epidermis, 3 random locations for each skin section and 4-5 sections for each biopsy were measured, and the average value was calculated. Expression of Ki-67, HB-EGF, p-ERK, and p-STAT3 was detected using rabbit antimouse Ki-67 antibody (1:400; catalog 9129; Cell Signaling Technology); goat anti-mouse HB-EGF antibody (1:400; catalog AF8239; R\&D Systems); rabbit anti-human p-STAT3 antibody (1:200; catalog 9131; Cell Signaling Technology); and rabbit anti-human p-ERK antibody (1:200; catalog 9101; Cell Signaling Technology). Matched IgG isotype controls were included for each staining. Primary antibodies were detected with Alexa Fluor 594-conjugated goat anti-rabbit IgG $(\mathrm{H}+\mathrm{L})$ or rabbit anti-goat IgG $(\mathrm{H}+\mathrm{L})$ (both from Life Technologies) and visualized with a fluorescence microscope (Zeiss). Infiltrating immune cells were characterized by immunofluorescence staining. Neutrophils, macrophages, and $\mathrm{T}$ cells were detected using rabbit anti-mouse Gr-1 (1:100; catalog 550291; BD Pharmingen); rabbit anti-mouse CD68 (1:10,000; catalog MCA1957; AbD Serotec); and rabbit anti-mouse CD4 (1:100; catalog 553647; BD Pharmingen). Matched IgG isotype controls were included for each staining. Primary antibodies were detected with biotinylated anti-rabbit IgG $(\mathrm{H}+\mathrm{L})$ (1:200; catalog BA-1000; Vector Laboratories) and then streptavidin Alexa Fluor 488 (1:500; catalog S-11223; Life Technologies). All slides were evaluated by 2 independent observers unaware of the identity of the biopsy.

Isolation and culture of mouse epidermal keratinocytes. The mouse epidermal skin was separated from the dermis after incubation with
Dispase II (Life Technologies) at $4^{\circ} \mathrm{C}$ for overnight and digested with $0.05 \%$ trypsin at $37^{\circ} \mathrm{C}$ for 10 minutes. Keratinocytes were collected and cultured in Medium 154CF (Cascade Biologics, Life Technologies) for 7 days, then treated with mouse TNF- $\alpha(50 \mathrm{ng} / \mathrm{ml}$; PeproTech) for 24 hours. RNA was extracted for qRT-PCR analysis.

Human ex vivo wound model. Human skin samples were obtained from routine abdominal or breast reduction surgeries. The wounds were made under sterile conditions with a $3-\mathrm{mm}$ biopsy punch on the epidermal side of the skin. The wounds were excised with a $6-\mathrm{mm}$ biopsy punch and subsequently transferred to a cell culture plate containing DMEM plus 10\% FBS and antibiotics (penicillin $50 \mathrm{U} / 1$ and streptomycin $50 \mathrm{mg} / \mathrm{ml}$; Life Technologies). In vivo LNA miR-132 inhibitor (anti-miR-132) or negative control (anti-miR-Ctrl) (0.5 nmol; Exiqon) was dissolved in 30\% pluronic F-127 gel (Sigma-Aldrich) and topically applied to the wounds immediately and 2 days after injury. Wound samples were collected 3 days after injury for histological and gene expression analyses.

Statistics. Statistical significance was determined by 2-tailed Student's $t$ test. The $P$ values for gene ontology enrichment analysis were calculated by Fisher's exact test for genes regulated by miR-132 in keratinocytes. A correlation between the expression of different genes in the same sample was made using Pearson's correlation test on log-transformed data. A $P$ value of less than 0.05 was considered statistically significant.

Study approval. Written informed consent was obtained from all healthy donors for the collection and use of clinical samples. The study was approved by the Stockholm Regional Ethics Committee and conducted according to Declaration of Helsinki principles. All procedures involving mice were approved by the North Stockholm ethics committee for the care and use of laboratory animals.

\section{Acknowledgments}

We wish to thank the healthy donors for their participation in this study. We thank Gangwen Han and Xiao-Jing Wang (University of Colorado, Denver, USA) for providing TGF- $\beta$ Tg mouse skin; Roger Rezzonico (Université Nice Sophia Antipolis, Nice, France), and Warangkana Lohcharoenkal, Stanley Cheuk, Ankit Srivastava, and Xintong Jiang (all from KI) for their fruitful discussions and suggestions; and Anna-Lena Kastman, Helena Griehsel, and Maria E. Lundqvist (all from KI) for their excellent technical support. We also thank the microarray core facility at the Novum Bioinformatics and Expression Analysis (BEA) facility, which is supported by the KI board of research and the Karolinska University Hospital research committee. Thanks are also due to Ulrike Teichmann for appropriate handling of the miR-132-KO mouse line. E. Erikci and K. Chowdhury were supported by the Max Planck Society. This work was supported by grants from the Swedish Research Council (Vetenskapsrådet, project numbers K2014-85X-22500-01-3 and and 2014-4-285), the La RochePosay Foundation, the Hedlunds Foundation, the Welander and Finsens Foundation, the Von Kantzow Foundation, the Strategic Research Programme in Diabetes, and KI.

Address correspondence to: Ning Xu Landén, Molecular Dermatology Research Group, Center for Molecular Medicine (CMM), L8:02, Department of Medicine, Karolinska Institutet, SE-17176 Stockholm, Sweden. Phone: 46.8.51772158; E-mail: ning.xu@ki.se. 
1. Reinke JM, Sorg H. Wound repair and regeneration. Eur Surg Res. 2012;49(1):35-43.

2. Strbo N, Yin N, Stojadinovic O. Innate and adaptive immune responses in wound epithelialization. Adv Wound Care (New Rochelle). 2014;3(7):492-501.

3. Roupé KM, Nybo M, Sjöbring U, Alberius $P$, Schmidtchen A, Sørensen OE. Injury is a major inducer of epidermal innate immune responses during wound healing. J Invest Dermatol. 2010;130(4):1167-1177.

4. Mustoe TA, O'Shaughnessy K, Kloeters O. Chronic wound pathogenesis and current treatment strategies: a unifying hypothesis. Plast Reconstr Surg. 2006;117(7 suppl):35S-41S.

5. Ambros V, Lee RC, Lavanway A, Williams PT, Jewell D. MicroRNAs and other tiny endogenous RNAs in C. elegans. Curr Biol. 2003;13(10):807-818.

6. Janssen HL, et al. Treatment of HCV infection by targeting microRNA. N Engl J Med. 2013;368(18):1685-1694.

7. Lai WF, Siu PM. MicroRNAs as regulators of cutaneous wound healing. J Biosci. 2014; 39(3):519-524.

8. Hua Z, et al. MiRNA-directed regulation of VEGF and other angiogenic factors under hypoxia. PLoS One. 2006;1:e116.

9. Sun CY, et al. miR-15a and miR-16 affect the angiogenesis of multiple myeloma by targeting VEGF. Carcinogenesis. 2013;34(2):426-435.

10. Biswas S, et al. Hypoxia inducible microRNA 210 attenuates keratinocyte proliferation and impairs closure in a murine model of ischemic wounds. Proc Natl Acad Sci U S A . 2010;107(15):6976-6981.

11. Meisgen F, et al. MiR-146a negatively regulates TLR2-induced inflammatory responses in keratinocytes. J Invest Dermatol. 2014; 134(7):1931-1940.

12. Ramirez H, Patel SB, Pastar I. The role of TGF $\beta$ signaling in wound epithelialization. Adv Wound Care (New Rochelle). 2014;3(7):482-491.

13. Han G, Williams CA, Salter K, Garl PJ, Li AG, Wang XJ. A role for TGF $\beta$ signaling in the pathogenesis of psoriasis. JInvest Dermatol. 2010;130(2):371-377.

14. Li AG, Wang D, Feng XH, Wang XJ. Latent TGF $\beta 1$ overexpression in keratinocytes results in a severe psoriasis-like skin disorder. EMBO J. 2004;23(8):1770-1781.

15. Lewis BP, Burge CB, Bartel DP. Conserved seed pairing, often flanked by adenosines, indicates that thousands of human genes are microRNA targets. Cell. 2005;120(1):15-20.

16. Alexiou P, Maragkakis M, Papadopoulos GL, Simmosis VA, Zhang L, Hatzigeorgiou AG. The DIANA-mirExTra web server: from gene expression data to microRNA function. PLoS One. 2010;5(2):e9171.

17. Huang da W, Sherman BT, Lempicki RA. Systematic and integrative analysis of large gene lists using DAVID bioinformatics resources. Nat Protoc. 2009;4(1):44-57.

18. Butcher EC. Leukocyte-endothelial cell recognition: three (or more) steps to specificity and diversity. Cell. 1991;67(6):1033-1036.

19. Murphy K. Janeway's Immunobiology. 8th ed. New York, New York, USA: Garland Science, Taylor \& Francis Group; 2012.

20. Subramanian A, et al. Gene set enrichment analysis: a knowledge-based approach for interpreting genome-wide expression profiles. Proc Natl Acad Sci U S A. 2005;102(43):15545-15550.

21. Gosselin K, Touzet H, Abbadie C. Rel/NF-kappaB target genes. Institut de Biologie de Lille et LIFL Web site. http://bioinfo.lifl.fr/NF-KB/. Updated February 1, 2004. Accessed May 26, 2015.

22. Pasparakis M. Regulation of tissue homeostasis by NF- $\mathrm{BB}$ signalling: implications for inflammatory diseases. Nat Rev Immunol. 2009;9(11):778-788.

23. Scholzen T, Gerdes J. The Ki-67 protein: from the known and the unknown. JCell Physiol. 2000;182(3):311-322.

24. Jorissen RN, Walker F, Pouliot N, Garrett TP, Ward CW, Burgess AW. Epidermal growth factor receptor: mechanisms of activation and signalling. Exp Cell Res. 2003;284(1):31-53.

25. Sanderson MP, Dempsey PJ, Dunbar AJ. Control of ErbB signaling through metalloprotease mediated ectodomain shedding of EGF-like factors. Growth Factors. 2006;24(2):121-136.

26. Molnár V, et al. MicroRNA-132 targets HB-EGF upon IgE-mediated activation in murine and human mast cells. Cell Mol Life Sci. 2012;69(5):793-808.

27. Stoll SW, Rittié L, Johnson JL, Elder JT. Heparin-binding EGF-like growth factor promotes epithelial-mesenchymal transition in human keratinocytes. J Invest Dermatol. 2012;132(9):2148-2157.

28. Ucar A, et al. The miRNA-212/132 family regulates both cardiac hypertrophy and cardiomyocyte autophagy. Nat Commun. 2012;3:1078.

29. Koh TJ, DiPietro LA. Inflammation and wound healing: the role of the macrophage. Expert Rev Mol Med. 2011;13:e23.

30. Heilborn JD, et al. The cathelicidin anti-microbial peptide LL-37 is involved in re-epithelialization of human skin wounds and is lacking in chronic ulcer epithelium. J Invest Dermatol. 2003;120(3):379-389.

31. Zhang S, et al. Downregulation of miR-132 by promoter methylation contributes to pancreatic cancer development. Carcinogenesis. 2011;32(8):1183-1189.
32. Jungert K, et al. Smad-Sp1 complexes mediate TGF $\beta$-induced early transcription of oncogenic Smad7 in pancreatic cancer cells. Carcinogenesis. 2006;27(12):2392-2401.

33. Wanet A, Tacheny A, Arnould T, Renard P. miR-212/132 expression and functions: within and beyond the neuronal compartment. Nucleic Acids Res. 2012;40(11):4742-4753.

34. Wang J, Xu G, Shen F, Kang Y. miR-132 targeting cyclin E1 suppresses cell proliferation in osteosarcoma cells. Tumour Biol. 2014;35(5):4859-4865.

35. Li S, et al. MicroRNA-132 is frequently down-regulated in ductal carcinoma in situ (DCIS) of breast and acts as a tumor suppressor by inhibiting cell proliferation. Pathol Res Pract. 2013;209(3):179-183.

36. Anand S, et al. MicroRNA-132-mediated loss of p120RasGAP activates the endothelium to facilitate pathological angiogenesis. Nat Med. 2010;16(8):909-914.

37. Sano S, Chan KS, DiGiovanni J. Impact of Stat3 activation upon skin biology: a dichotomy of its role between homeostasis and diseases. J Dermatol Sci. 2008;50(1):1-14.

38. Sebolt-Leopold JS, Herrera R. Targeting the mitogen-activated protein kinase cascade to treat cancer. Nat Rev Cancer. 2004;4(12):937-947.

39. Knudsen SL, Mac AS, Henriksen L, van Deurs B, Grøvdal LM. EGFR signaling patterns are regulated by its different ligands. Growth Factors. 2014;32(5):155-163.

40. Stoll SW, et al. Metalloproteinase-mediated, context-dependent function of amphiregulin and HB-EGF in human keratinocytes and skin. J Invest Dermatol. 2010;130(1):295-304.

41. Roepstorff K, et al. Differential effects of EGFR ligands on endocytic sorting of the receptor. Traffic. 2009;10(8):1115-1127.

42. Poumay Y, de Rouvroit CL. HB-EGF, the growth factor that accelerates keratinocyte migration, but slows proliferation. J Invest Dermatol. 2012;132(9):2129-2130.

43. Nakahama T, et al. Aryl hydrocarbon receptormediated induction of the microRNA-132/212 cluster promotes interleukin-17-producing T-helper cell differentiation. Proc Natl Acad Sci US A. 2013;110(29):11964-11969.

44. Xu N, et al. MiR-125b, a microRNA downregulated in psoriasis, modulates keratinocyte proliferation by targeting FGFR2. J Invest Dermatol. 2011;131(7):1521-1529.

45. Edgar R, Domrachev M, Lash AE. Gene Expression Omnibus: NCBI gene expression and hybridization array data repository. Nucleic Acids Res. 2002;30(1):207-210.

46. Botusan IR, et al. Stabilization of HIF-1 $\alpha$ is critical to improve wound healing in diabetic mice. Proc Natl Acad Sci U S A. 2008;105(49):19426-19431. 